\title{
Prediction of Thrombus Formation in Ladder Network and Stability of Thrombus in a Bifurcation
}

\author{
Hari Hara Sudhan Lakshmanan
}

Follow this and additional works at: https://researchrepository.wvu.edu/etd

\section{Recommended Citation}

Lakshmanan, Hari Hara Sudhan, "Prediction of Thrombus Formation in Ladder Network and Stability of Thrombus in a Bifurcation" (2018). Graduate Theses, Dissertations, and Problem Reports. 6028. https://researchrepository.wvu.edu/etd/6028

This Thesis is protected by copyright and/or related rights. It has been brought to you by the The Research Repository @ WVU with permission from the rights-holder(s). You are free to use this Thesis in any way that is permitted by the copyright and related rights legislation that applies to your use. For other uses you must obtain permission from the rights-holder(s) directly, unless additional rights are indicated by a Creative Commons license in the record and/ or on the work itself. This Thesis has been accepted for inclusion in WVU Graduate Theses, Dissertations, and Problem Reports collection by an authorized administrator of The Research Repository @ WVU. For more information, please contact researchrepository@mail.wvu.edu. 


\title{
Prediction of Thrombus Formation in Ladder Network and Stability of Thrombus in a Bifurcation
}

\author{
Hari Hara Sudhan Lakshmanan \\ Thesis submitted to the \\ Benjamin M. Statler College of Engineering and Mineral Resources \\ at West Virginia University \\ in partial fulfillment of the requirements \\ for the degree of
}

Master of Science in

Chemical Engineering

Jeevan Maddala, Ph.D., Chair Debangsu Bhattacharyya, Ph.D., Fernando Lima, Ph.D.

Department of Chemical and Biomedical Engineering

$$
\begin{gathered}
\text { Morgantown, West Virginia } \\
\text { January } 262018
\end{gathered}
$$

Keywords: Microfluidic ladder network, Thrombus, Shear rate, Critical bifurcation ratio, Thrombin Copyright 2018 Hari Hara Sudhan Lakshmanan 


\begin{abstract}
Prediction of Thrombus Formation in Ladder Network and Stability of Thrombus in a Bifurcation
\end{abstract}

Hari Hara Sudhan Lakshmanan

Thrombus formation on complex branched networks like extracorporeal membrane oxygenators (ECMO) is poorly understood. In this work, we built a COMSOL model on branched networks, to understand and predict the thrombus formation under blood flow. A microfluidic ladder network design was used to study the change in hemodynamics. The microfluidic ladder sizes are chosen to mimic the physiologically relevant shear rates of $300 \mathrm{~s}^{-1}$ and $700 \mathrm{~s}^{-1}$ in main channels and bypasses respectively. The COMSOL model results were analyzed to understand the flow dynamics inside the ladder network. We predicted the thrombus nucleation points in the ladder network, based on shear grad ients, velocity profile, thrombin transport and platelet concentration. Thrombi were modeled as 2D spheres in the predicted locations of the ladder network. Thrombus growth was modeled in six cases to account for the progressive growth of thrombi the ladder network. Shear rates, thrombin transport rate and platelet percentage were estimated for each case of simulation from COMSOL to develop a set of metrics for thrombus prediction. The predictions from the model were then validated using experimental data from a similar microfluidic ladder device. Experiments were done using recalcified whole human blood pumped into the device at a constant inlet flow rate of $2 \mu \mathrm{L} \mathrm{min}{ }^{-1}$. Observations from the experiment revealed that clots are formed at the intersection of bypasses with main channels and not all clots were occlusive. The formation of thrombus followed a pattern progressing from $n^{\text {th }}$ bypass to $(n+1)^{\text {th }}$ bypass. The geometry induced effects on thrombus formation patterns proved the hypothesis that geometry plays a vital role in spatio-temporal aspects of thrombus nucleation and growth. Images recorded in the experiments of ladder network revealed that not all thrombi in the ladder network went to complete occlusion. Hydrodynamic forces were found to control the occlusion scenario of the thrombus. Threshold of occlusion was studied in a thrombus growing in a straight channel and the results were extended to bifurcations - blood vessels branching into two smaller vessels. Our study revealed that bifurcation ratio and the occlusion of thrombus in a bifurcation are related to each other. Mathematical model developed to understand the critical bifurcation ratios can be used to study the interaction of multiple clots in a geometry from stability perspective. An integrated model that predicts thrombus nucleation and stability in a given geometry holds potential in development of therapeutic and diagnostic devices for blood disorders. 


\section{Acknowledgement}

I thank my advisor Dr.Jeevan Maddala for his expertise, patience, and steadfast assistance with this work. Without his support and guidance none of this would have been possible. I also thank the support of my father Lakshmanan, mother Deivanai and sisters Meenakshi and Lakshmi who have helped me through all the challenges in life.

I also thank my committee members Dr.Debangsu Bhattacharyya and Dr.Fernando Lima for their valuable comments and advice. Special thanks to Dr. Debangsu Bhattacharyya for allowing the use of his lab space for research.

I thank Dr.Owen McCarty and Jevgenia Zilberman from the Department of Biomedical Engineering at Oregon Health and Science University for their valuable support during this work.

I thank my friends, Srikanth, Dr.Jothi Ganesh, Jayaram Subramanian, Irfan, Sarojini, Pushpitha, Paul Akula and Keshav for their words of encouragement and motivation during the research.

Funding for this research came from West Virginia University startup funds awarded to Dr.Jeevan Maddala. 


\section{TABLE OF CONTENTS}

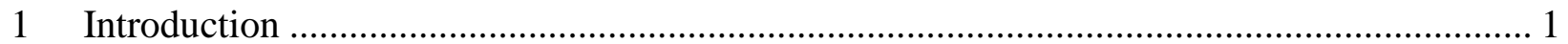

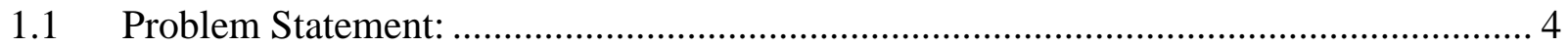

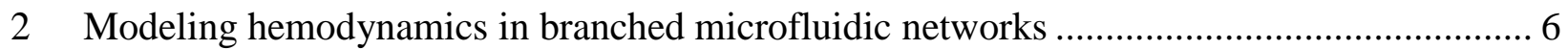

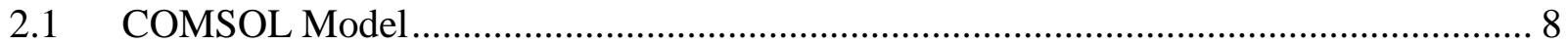

2.2 Nucleation Points from Simulation .................................................................... 14

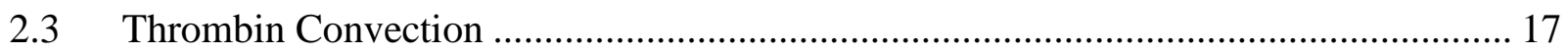

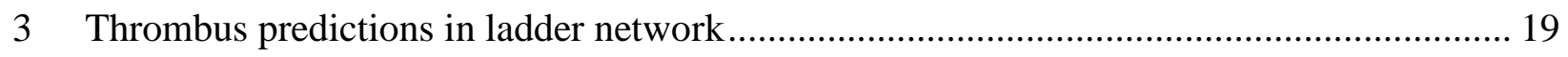

3.1 Metrics to Determine Thrombus Formation .................................................................. 19

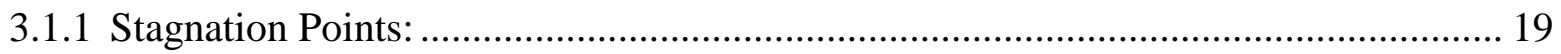

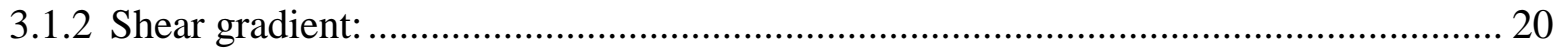

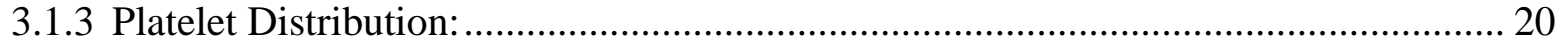

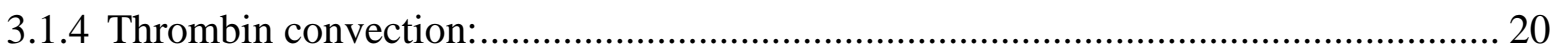

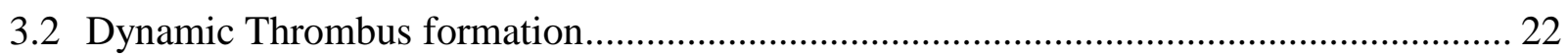

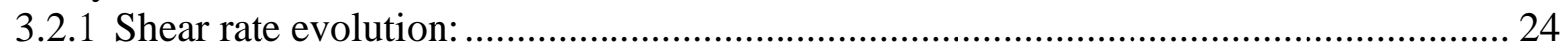

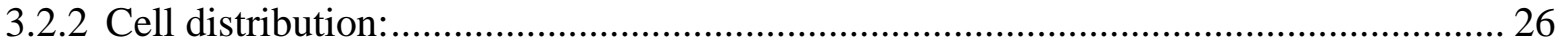

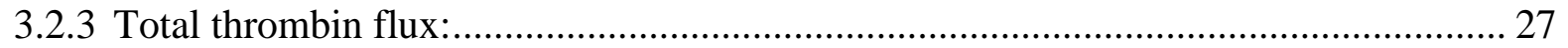

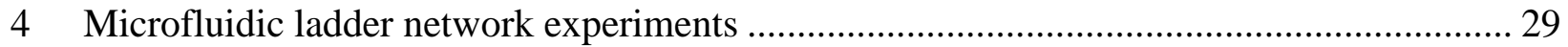

4.1 Fabrication of microfluidic ladder network ………................................................... 29

4.2 Thrombus formation in Microfluidic Ladder Network ............................................. 31

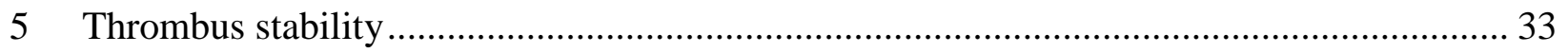

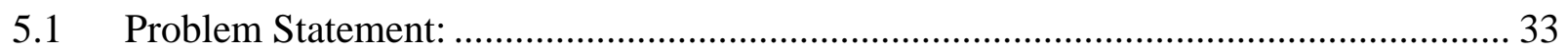

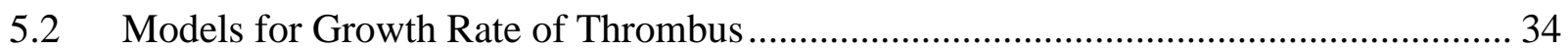

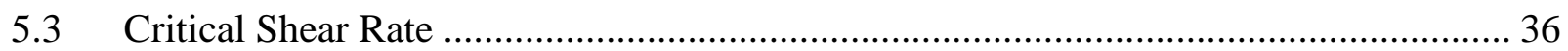

5.4 Shear rate analysis in a Straight Channel ................................................................... 37

5.4.1 Constant Flow Rate ………………………………………............................. 39

5.4.2 Constant Pressure Drop.................................................................................. 40

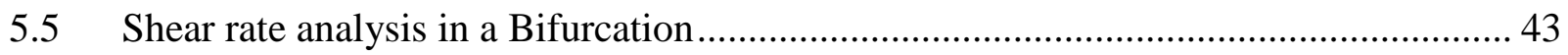

5.5.1 Model Development ………………………….................................................... 45

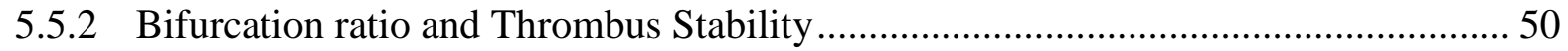

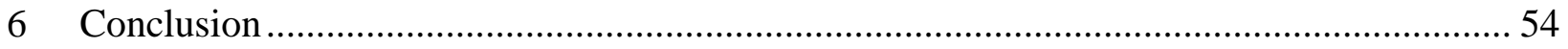

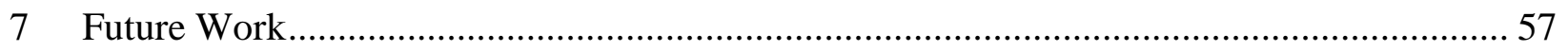

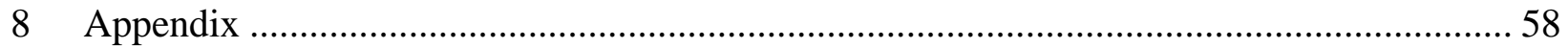

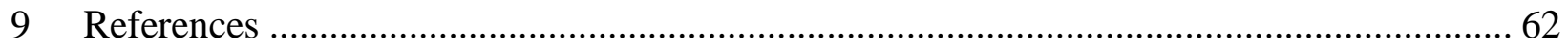




\section{LIST OF FIGURES}

Figure 1 (a) Branched network of blood vessels (b) Network of blood vessels with thrombi ....... 4 Figure 2 Design parameters of a multi-bypass ladder network with ten bypasses and two main channels

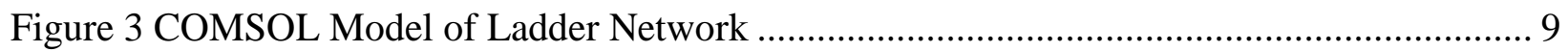

Figure 4 Platelet Margination at the inlet of ladder network- COMSOL .................................... 13

Figure 5 Shear rate and Streamlines plot of blood flow in ladder network-COMSOL ................. 15

Figure 6 Velocity Field of blood in ladder network-COMSOL .............................................. 16

Figure 7 Trajectories of platelets and RBCs in ladder network ............................................. 17

Figure 8 2D Surface plot of Total thrombin flux in ladder network from COMSOL; Red regions in the plot signify higher thrombin flux, blue regions are the regions with lowest thrombin flux. Flux seen in the plot is higher along the streamlines showing the convection dominated thrombin

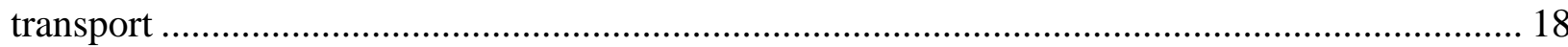

Figure 9 Modeling approach to prediction of thrombus formation patterns................................ 21 Figure 10 Thrombus evolution dynamics in the ladder network. a) Case 1 bypass I 5\% occlusion; b) Bypass I 50\% and bypass II 5\% occlusion; c) Bypass I 100\%, bypass II 50\% and bypass III 5\% occlusion; d) Bypass I, II 100\%, bypass III 50\% and bypass IV 5\% occlusion; e) Bypass I,II, III, 100\%, bypass IV 50\% and bypass V 5\% occlusion; f) Bypass I,II,III and IV 100\% and

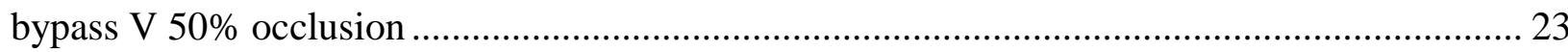

Figure 11 Occlusion Pattern simulated in COMSOL for bypass 1 to 5 . Each set of thrombi in $(\mathrm{n}+1)$ th bypass move from $0 \%$ occlusion to $100 \%$ occlusion in 15 minutes after the thrombi in the nth bypass.

Figure 12 Cut Line method for measuring (a) Total thrombin flux, (b) Percentage of platelets in

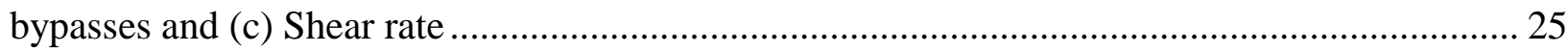

Figure 13 Maximum shear rate in bypasses at all the stages of thrombus progression. ............... 26

Figure 14 Effect of thrombus formation on the number of platelets in bypasses......................... 27

Figure 15 Rate of thrombin transport from each bypass. Thrombin transport increases with

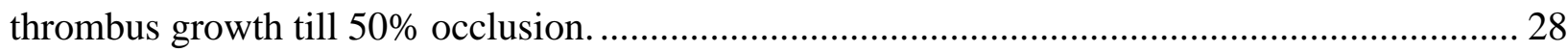

Figure 16 (a) Microfluidic ladder network as seen under a Differential Interference Contrast (DIC) microscope (b) Experimental setup of the ladder network with the blood pumped through

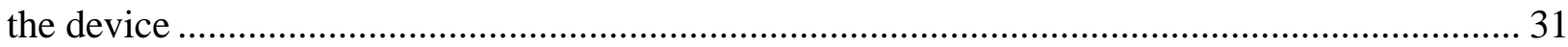

Figure 17 Experimental images of Thrombus in microfluidic Ladder network. .......................... 32 Figure 18 Thrombus Stability in different geometries- Which thrombus will occlude the vessel ?

Figure 19 Thrombus growth in a straight channel, $\mathrm{D}_{0}$ is the initial diameter of the channel, $\mathrm{D}_{2}$ is the open diameter at any instant during thrombus growth, $1_{2}$ is the length of thrombus. Pressure Drop is analgous to Potential difference and flow rate is analgous to current in an electrical

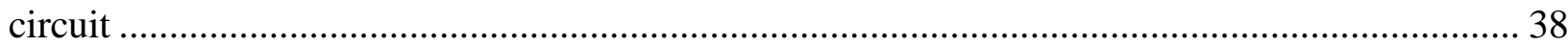

Figure 20 Shear rate on the thrombus in a straight channel under constant flow rate .................. 40 
Figure 21 Shear rate on the thrombus in a straight channel under constant pressure drop.....

Figure 22 Maximum Shear rate dependency on thrombus length ratio;. 1/L > 0.5 changes the shear rate behavior from non-linear to monotonically decreasing.... 42 Figure 23 Parent vessel of diameter D bifurcating into two daughter branches with diameters $D_{1}$ and $\mathrm{D}_{2}$ 43 Figure 24 a) Bifurcation as a network of hydraulic resistances, Branch 2 as a single resistance $\mathrm{R}_{2}$ and the inlet channel resistance $\mathrm{R}$. (b) Branch 1 is the sum of three reistances, $\mathrm{R}_{1}$ ', $\mathrm{R}_{1}$ ', $\mathrm{R}_{1}$ ', , where $\mathrm{R}_{1}{ }^{\prime}$ is the thrombus......................................................................................... 46 Figure 25 Bifurcation ratios $\mathrm{r}_{1}, \mathrm{r}_{2}$ and Shear ratio, $\gamma \max / \gamma c$ plot for $\emptyset=0.021$ and an inlet diameter $\mathrm{D}=38 \mu \mathrm{m}$ 


\section{Introduction}

Thrombosis is the obstruction of blood flow in the circulatory system by a thrombus/blood clot in the blood vessel. A thrombus is a cellular aggregate of platelets and Red Blood Cells (RBCs) bound together by fibrin. When an artery or vein is injured, a thrombus is formed on the surface of the injury to prevent the leakage of blood from the blood vessel. This phenomenon is known as hemostasis.

Sometimes a portion of the thrombus could dislodge from the injury due to the hydrodynamic forces. The dislodged thrombus portion travelling in the bloodstream is called an embolus. When this embolus encounters a blood vessel smaller than itself, it blocks the flow of blood into the vessel, this phenomenon is termed as embolism. Embolism in lung arteries is known as pulmonary embolism, a serious medical condition that can cause death.

A completely occluding blood clot in a blood vessel could cause Cardiac arrest, Stroke, Paralysis and Deep Vein Thrombosis (DVT) based on the location of the vessel in the human body. Cardiac diseases alone are responsible for every 1 in 4 deaths in the United States of America as per NIH data. Cardiac arrest occurs when a part of the blood vessel leading to or from the heart is blocked by a thrombus. This cuts off the blood flow to the part of the heart muscle supplied blood by the artery. When this condition prolongs, the heart muscles in that area die leading to either permanent muscle damage or death. American Heart Association (AHA) 2015 heart and stroke statistics shows that heart disease is the No. 1 cause of death

globally, with 17.3 million deaths each year. As per the predictions, this number is expected to increase to 23.6 million by 2030 . 
Stroke is the phenomenon of blocking blood flow in the brain vascular network due to a thrombus. Stoke could cause paralysis, loss of vision, and in serious cases death. Stroke is the fifth leading cause of death and costs USA $\$ 34$ billion annually, as per the data from Center for Disease Control and Prevention (CDC). According to World Health Organization, 15 million people suffer stroke worldwide every year, of which one third suffer death. Patients suffer serious mobility disorder post stroke. Stroke and Cardiac arrest are some of the most serious disorders caused by thrombosis.

When the integrity of a blood vessel is disrupted due to an injury, the sub-endothelium of vessels with collagen fibers and tissue factor (TF) are exposed to platelets flowing in the blood. The platelets that come in contact with the collagen fibers undergo a set of events termed as 'activation' (Harmening, 2002). The primary events in the activation of platelet are shape change, aggregation of platelets and catalyzation of thrombin generation from blood - another pro-coagulant that can activate platelets. This process of a platelet activating other platelets through supporting thrombin generation is known as 'platelet recruitment' in medical terms. The activation of platelets through collagen is called 'extrinsic pathway' of thrombus formation. During the formation of thrombus some of the RBCs flowing in blood are also bound together with the platelets. The aggregate of platelets formed over the injury site is stabilized by the fibrin strands generated from fibrinogen- a protein found in blood.

Activation of platelets occurs either due to thrombin in blood from the surface catalysis of platelets, tissue factor in sub-endothelium or shear forces that can activate platelets to catalyze thrombin generation. Thrombus formation in-vivo involves multitude of chemical and 
biological reactions and cellular interactions. It is difficult to control and isolate each factor that is responsible for thrombus formation. Ever since the development of microfluidic flow chamber, researchers have found it useful to apply microfluidic devices in the study of thrombosis and hemostasis in-vitro (Colace, G.W.Tormoen, O.J.T.McCarty, \& S.L.Diamond, 2013).

Studies conducted in flow chambers offer crucial information on the biophysical aspects of thrombosis. In flow chambers, one can study thrombus formation under isolated shear rates, with a power to understand arterial and venous thrombus formation under different conditions. Microfluidic channels can be fabricated in different dimensions as needed for the research. The application of microfluidic devices are demonstrated in studying hydrodynamic interactions in thrombus growth and diagnosing clotting disorders through controlled studies. Experiments conducted using flow chambers have uncovered the various pathways of platelet activation and thrombus formation leading to development of innovative drugs to treat thrombosis.

Microfluidic devices did not stop at labs but are moving into the hospitals for treatment and diagnostics. One such important applications is the microfluidic Extracorporeal Oxygenators (ECMOs). ECMOs are devices used to remove $\mathrm{CO}_{2}$ from blood and oxygenate $\mathrm{RBCs}$ artificially outside the body. ECMOs are used on patients with cardiac and respiratory disorders that make it impossible for the body to maintain enough oxygen levels in blood (Wu, 2013).

Design of ECMOs is inspired from the design of human vasculature itself, and involves branched network of channels placed adjacent to each other. These extracorporeal devices face frequent clotting problem and necessitate the usage of heparin -an anti-coagulant- to 
suppress clot formation (Boyer \& Swartz, 1991). Use of heparin carries with itself myriad side effects that could create long term blood clotting disorders. The focus of the study in this thesis is to understand and predict the effects of geometry on thrombus nucleation, growth and stability.

\subsection{Problem Statement:}

Vasculature is a complex network of blood vessels branching out into different sizes. In this study, the focus was to answer the following questions pertaining thrombosis and geometry. Figure 1 is an example of a branched network of blood vessels. The following questions relevant to thrombosis form the basis of this study.

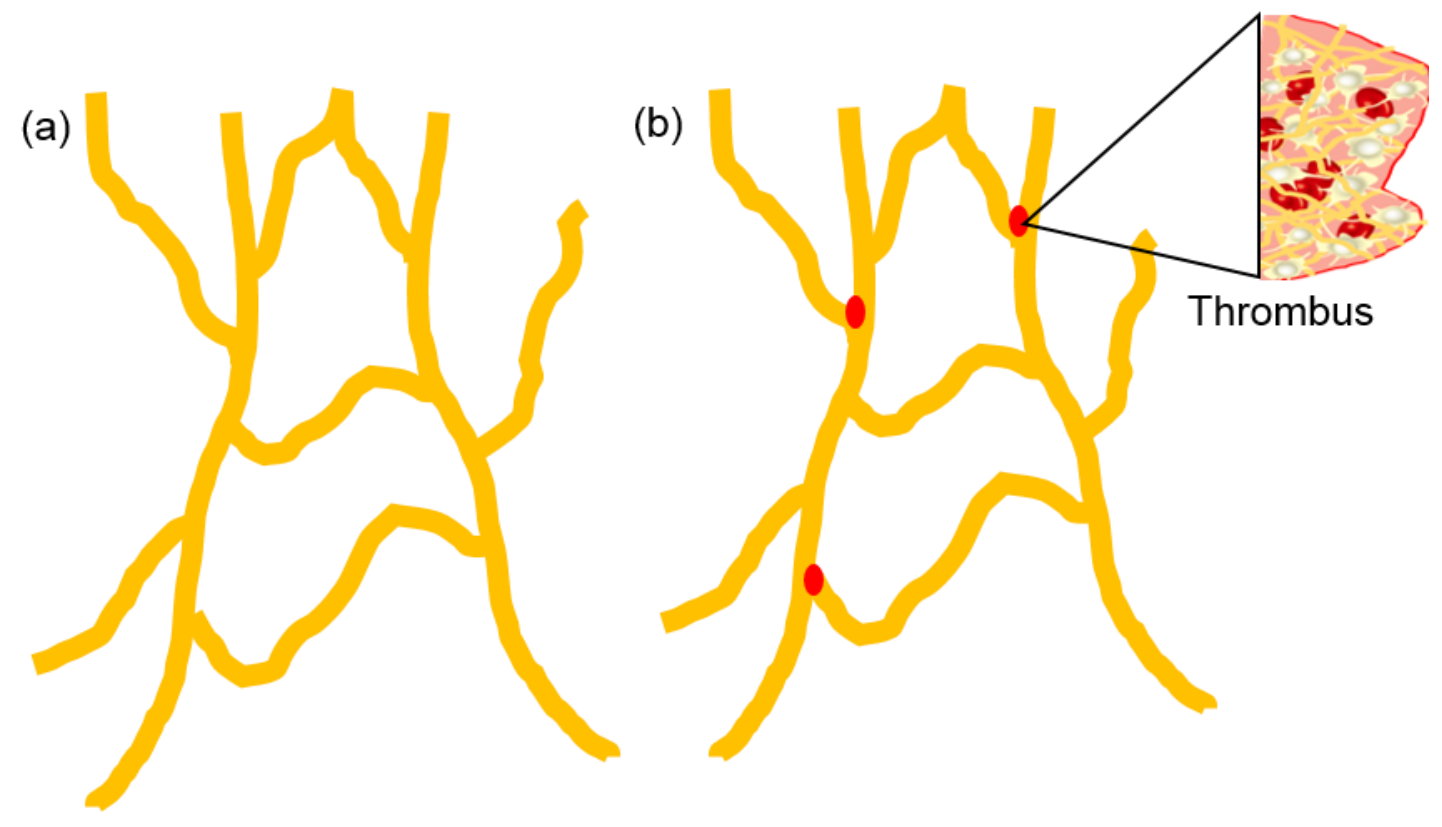

Figure 1 (a) Branched network of blood vessels (b) Network of blood vessels with thrombi

In a network as shown in Figure 1 (a), where will a thrombus form? What are the factors that favor an $(\mathrm{x}, \mathrm{y})$ to be the nucleation point of a thrombus over other locations? Figure 1 (b) shows the 
vascular network with the thrombi as red circles in the network. The next question concerns the stability of thrombus in terms of occlusion. A thrombus is considered stable if it does not grow to completely occlude the blood vessel. Which of the thrombi in Figure 1 (b) will grow to occlude the blood vessel?

The first part of the thesis, chapters $2-4$, focuses on the prediction of thrombus formation in a branched network through computational fluid dynamics model developed in COMSOL, validation of results from the model with experimental data and establishing a relation between geometry and thrombus formation. The second part of the thesis in chapter 5, focuses on investigating the geometrical dependence of thrombus stability with respect to occlusion. Theory of thrombus stability was built up from thrombus growing in straight channels to thrombus in bifurcations. A mathematical model was developed using first principles to identify the importance of geometry in the occlusion of thrombus. In the next chapter we discuss the modeling of ladder network, a geometry that was used in this work to predict thrombus formation. 


\section{Modeling hemodynamics in branched microfluidic networks}

A microfluidic ladder network with ten bypasses was used as a representative of a branched system of channels of varying sizes Figure 2. The ladder network is also very similar to the microfluidic ECMO developed for infants (Wu, 2013). Current studies on thrombosis focus on single microfluidic channel which does not bring out many geometrical factors that could affect thrombus formation. Ladder network brings out multitude of shear rates in a single device with the presence of stagnation points and recirculation zones.

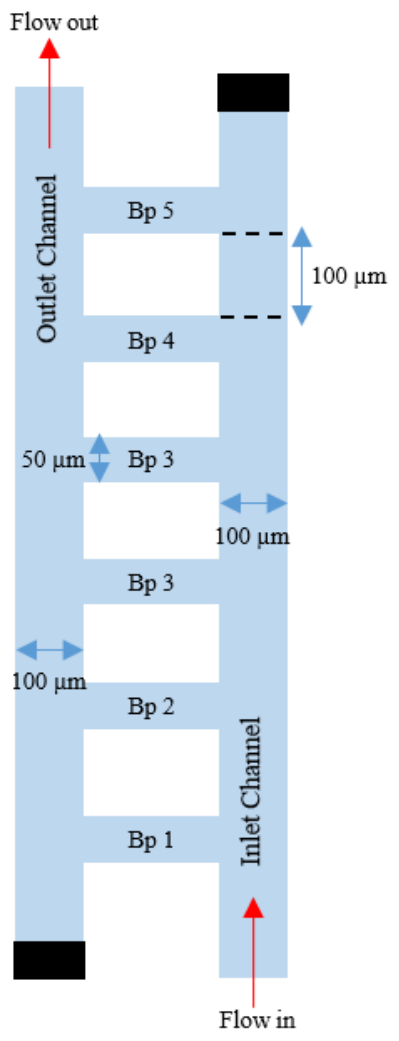

Figure 2 Design parameters of a multi-bypass ladder network with ten bypasses and two main channels

The purpose of this research to predict the nucleation points of thrombus in ladder network through a model developed in COMSOL. This will add to the existing literature an approach with an integrated model that can be used to assess extracorporeal devices on their 
thrombogenicity. Current models in literature are more focused on modeling the growth of individual thrombus and so they cannot predict the thrombus formation patterns in a network such as ladder. The existing information on triggers of thrombus formation have been integrated in this study to create an integrated model to identify thrombus nucleation in ladder network.

Computational fluid dynamic methods are widely used to understand the flow of fluids in microfluidic devices. Any given geometry, is discretized into nodes, whose sizes depend on the physics involved. Partial Differential Equations (PDE) involving pressure, viscosity, density and velocity are solved in those nodes with the chosen boundary conditions. In this study, a 2D model of ladder network was developed in COMSOL environment. COMSOL, a commercial finite element software, offers flexibility in terms of coupling and solving multiple physics involved in the flow together. This is essential as our study involves the physics of flow of fluids, particles and dilute species. Simulations were performed in COMSOL with the following three modules, 1. Creeping flow 2. Particle tracing for fluid flow and Transport of Diluted Species.

All the parameters such as viscosity, density, diameters of particles and other constants were defined under global definitions. Variables under global definitions can be used in any module or results section of the simulation.

Creeping flow module solves Navier stokes equation for Newtonian fluid flow and equations of motion for Non-newtonian fluid flows. Boundary conditions at the inlet, outlet and walls of the geometry are primary to solve the PDEs involved in flow description. For nonnewtonian fluids, this module offers Power law, Carreau model and a User defined option. 
Particle tracing for fluid flow module computes the trajectory of particles flowing in a fluid including particle-fluid interactions. This module allows the use of Newtonian, Lagrangian or Hamiltonian formulations for particles. Pre-defined inlet distributions can be modeled according to the user requirements in this module. This feature is exploited in modeling the platelets distribution in the COMSOL model. Lot of complex forces on the particles can be modeled using the module.

Transport of diluted species module involves modeling the mass transfer of species dissolved in a fluid. It allows for the study of both steady state and time dependent mass transfer of dissolved species. Diffusion co-efficient can be modeled as a user-defined function that depends on direction.

\subsection{COMSOL Model}

We developed a 2D COMSOL model of a ladder network with two main channels of $100-\mu \mathrm{m}$ width, $100-\mu \mathrm{m}$ height and ten bypasses each of $50-\mu \mathrm{m}$ width and $100-\mu \mathrm{m}$ height. The bypasses are the bifurcations of main inlet channel into the outlet channel. The inlet and outlet channels are contralateral to each other.

We assumed blood to be fluid of constant density $1060 \mathrm{~kg} / \mathrm{m}^{3}$ with a constant inlet flow rate of $2 \mu \mathrm{L} \mathrm{min} \operatorname{mon}^{-1}$ through the inlet. The flow rate was chosen to mimic the physiological shear rates found in human microvasculature. At these low flow rates, $\operatorname{Re}<<1$, the flow is viscous dominated, and can be modeled as creeping flow.

$$
R e=\frac{\text { Inertial force }}{\text { Viscous force }}=\frac{\text { Du }}{\mu} \ll 1
$$

Equation of continuity is the conservation of mass that must be satisfied in all flow situations and thus forms the first equation for the flow model (1). 


$$
\frac{\partial \rho}{\partial t}+\nabla \cdot(\rho u)=0
$$

Flow of blood is an incompressible steady flow, which simplifies the equation (1) to

$$
\nabla \cdot u=0
$$

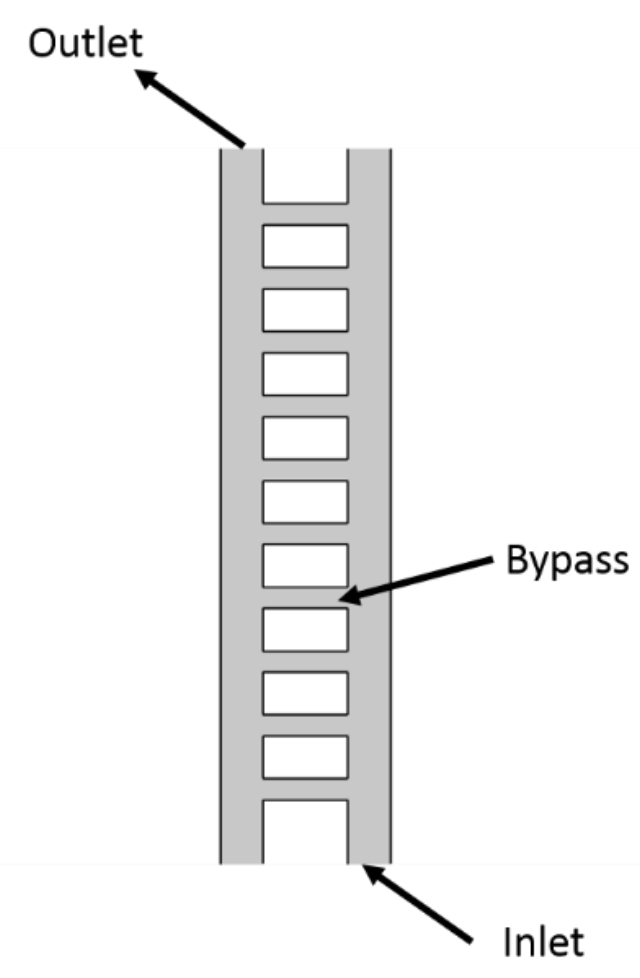

Figure 3 COMSOL Model of Ladder Network

Equation of motion describes the velocity and pressure of a fluid at every point in a flow system. Equation of motion in for fluid under flow is given by equation (3) (R.B., Stewart, \& E.N.Lightfoot, 1960) 


$$
\rho \frac{D(u(x, y))}{D t}=-\nabla \mathrm{P}+\nabla \cdot\left(\mu\left(\nabla \mathrm{u}+(\nabla \mathrm{u})^{T}\right)\right)
$$

$\frac{D}{D t}=\frac{\partial}{\partial t}+\frac{\partial}{\partial x}+\frac{\partial}{\partial y}-$ Substantial time derivative for two dimensions

$\mathrm{P}$ - Pressure,

$\rho$ - Density of fluid

$\mu$ - Viscosity,

t- Time,

$\mathrm{u}-$ Velocity

For creeping flow under steady state, the advection terms on the left hand side go to zero and the equation (3) simplifies to

$$
-\nabla \mathrm{P}+\nabla \cdot\left(\mu\left(\nabla \mathrm{u}+(\nabla \mathrm{u})^{T}\right)\right)=0
$$

Blood is a non-newtonian shear thinning fluid. The viscosity of blood decreases with increasing shear rate. Various mathematical models are found in literature that describes the viscosity of a non-newtonian shear thinning fluid. However, the power law model gives good fit for viscosity estimation of blood in the shear rates under consideration in this study (Hussain, 1999). Power law model equations were solved simultaneously along with equations of motion to estimate velocity, shear rate, viscosity and pressure at each node in the system.

The power law model for viscosity is given by the following equations

$$
\mu=\lambda|\dot{\gamma}|^{n-1}
$$




$$
\begin{aligned}
& \lambda(\dot{\gamma})=\mu_{\infty}+\Delta \mu \exp \left[-\left(1+\frac{|\dot{\gamma}|}{a}\right) \exp \left(\frac{-b}{|\dot{\gamma}|}\right)\right] \\
& n(\dot{\gamma})=n_{\infty}+\Delta n \exp \left[-\left(1+\frac{|\dot{\gamma}|}{c}\right) \exp \left(\frac{-d}{|\dot{\gamma}|}\right)\right]
\end{aligned}
$$

$$
\begin{aligned}
& \mu_{\infty}=0.035 \mathrm{~N} \mathrm{~s} \mathrm{~m}^{-2}, \\
& n_{\infty}=1.0, \\
& \Delta \mu=0.25 \mathrm{~N} \mathrm{~s} \mathrm{~m}^{-2}, \\
& \Delta n=0.45, \\
& \mathrm{a}=50 \mathrm{~s}^{-1}, \\
& \mathrm{~b}=3 \mathrm{~s}^{-1}, \\
& \mathrm{c}=50 \mathrm{~s}^{-1}, \\
& \mathrm{~d}=4 \mathrm{~s}^{-1}, \\
& \dot{\gamma}-\text { shear rate }
\end{aligned}
$$

$$
\dot{\gamma}=\frac{1}{2}\left[\nabla \mathrm{u}+(\nabla \mathrm{u})^{T}\right]
$$

$\nabla \mathrm{u}-$ Velocity gradient

Boundary conditions for the model are

1. Constant inlet flow rate

2. Constant outlet pressure

3. No slip at the walls 
These boundary conditions are in accordance with the experimental conditions to validate the results of simulation. The equations (1)-(8) are solved under creeping flow module that describes the flow of blood in the network. In order to predict the sites with high probability of thrombus formation we also needed to model the flow of major particles, platelets and RBCs in the system.

To account for platelets and RBCs that are critical to identifying thrombus formation we make use of particle tracing module in COMSOL. Platelets in blood prefer to occupy the 2-5 $\mu \mathrm{m}$ region closer to the wall. The bulkier RBCs push the platelets to the edge and stay in the core of blood. This phenomenon is called platelet margination. In order to capture the positions of platelets and RBCs in the device it is essential that we model the margination phenomenon. It is assumed that the platelets and RBCs are well marginated before they enter the device. In COMSOL one can pre-define the inlet distribution of particles by setting the density of particles proportional to a specific mathematical function that describes the particle inlet positions.

At the inlet, the RBCs are released with a normal distribution at the channel's center with a variance of $50 \mu \mathrm{m}$.

$$
\left.\rho \propto \exp \left(\left(\frac{-\left(x-350 \times 10^{-6}\right)}{10^{-3}}\right)\right)^{2}\right)
$$

$x-$ ' $\mathrm{x}$ ' co-ordinate defining position.

$x=350 \times 10^{-6}$ - center of the inlet channel

The platelets should occupy positions closer to the two walls of the inlet. This was achieved by the addition of two Gaussian distribution functions with means at both the walls. 


$$
\rho \propto \exp \left(\left(\frac{-\left(x-300 \times 10^{-6}\right)}{10^{-5}}\right)^{2}\right)+\exp \left(\left(\frac{-\left(x-400 \times 10^{-6}\right)}{10^{-5}}\right)^{2}\right)
$$

$x=350 \times 10^{-6}$ and $400 \times 10^{-6}$ are the walls of inlet channel

The final distribution of particles at the inlet is shown in Figure 4 with platelets (blue circles) and RBCs (red circles) marginated at the inlet.

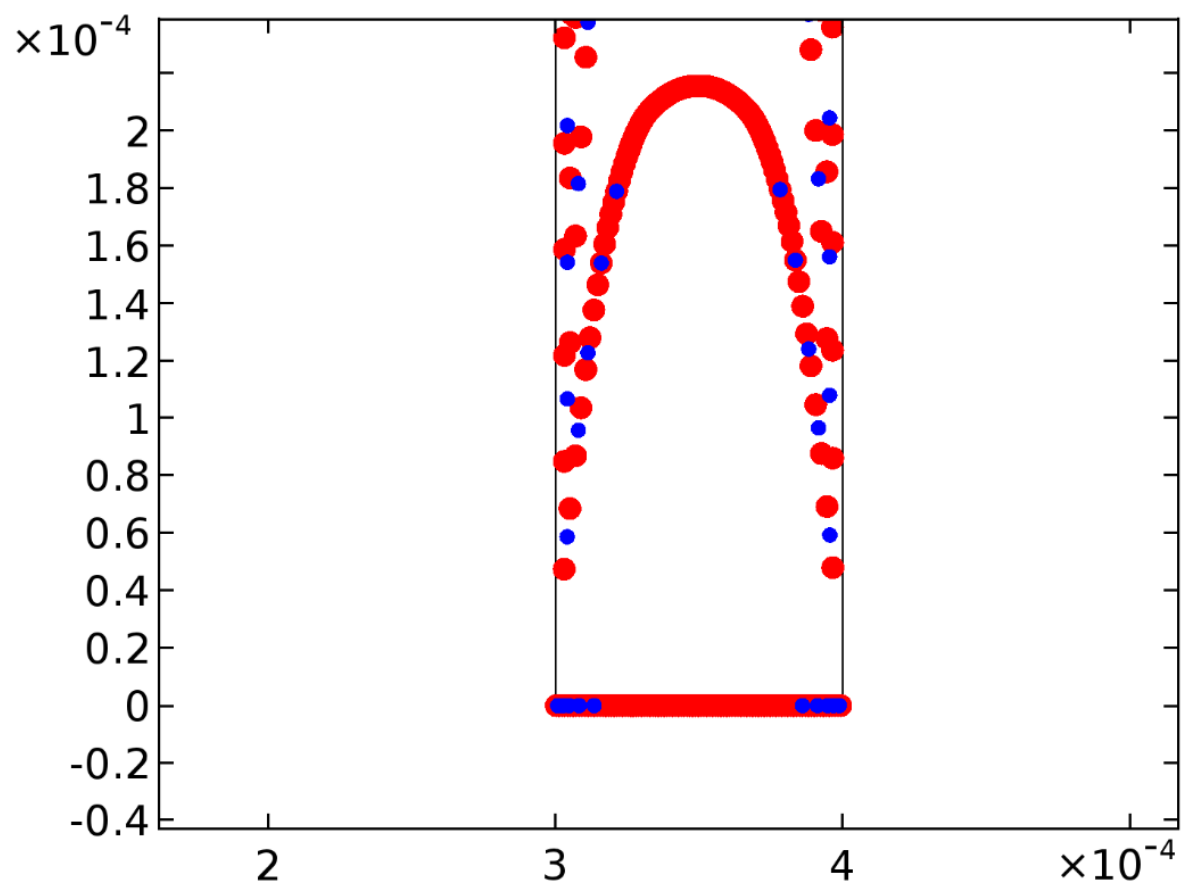

Figure 4 Platelet Margination at the inlet of ladder network- COMSOL

The number of platelets and RBCs in simulations were chosen based on the average hematocrit of human blood. Hematocrit is the percentage of volume of blood occupied by RBCs, and indicates the number of RBCs in blood sample. We assumed a hematocrit of 0.5 , i.e. $50 \%$ of the core of blood volume was assumed to be filled with RBCs. RBCs and platelets are assumed to be spheres of $8 \mu \mathrm{m}$ and $1.8 \mu \mathrm{m}$ with a density of $1060 \mathrm{~kg} / \mathrm{m}^{3}$. The particles are assumed to enter with 'zero velocity' at the inlet and are released every 0.05 seconds for 10 
seconds. Steady state of particle positions was reached at the end of 10 seconds. Motion of particles inside the ladder network was purely based on the viscous drag force imparted on the particle by the fluid.

The stokes law drag force was modeled in particle tracing for fluid flow module by the following equation

$$
F=\frac{18 \mu}{\rho_{p} d_{p}^{2}} m_{p} V_{r e l}
$$

$\mathrm{F}-$ drag force

$\rho_{p}$-particle density

$d_{p}$ - diameter of particle

$m_{p}-$ mass of particle,

$V_{\text {rel }}-$ relative velocity

Solutions to particle positions depends on the solutions from creeping flow module because of the relative velocity component involved in the drag force. Steady state solution obtained from creeping flow module is used as the basis for particle tracing module. Particle trajectory plot shows the position of platelets and RBCs at every time instant in the ladder network.

\subsection{Nucleation Points from Simulation}

Unsteady state simulations were performed to understand the particle trajectories and steady state simulations for the flow profiles inside the ladder network. The most probable sites for thrombus nucleation were predicted based on blood flow velocity, shear rate and blood cell 
trajectories. If the platelets spend more time at a point in the geometry, then the point can be expected to nucleate a thrombus. Streamlines of velocity indicate the separation of flow occurring near the intersection of bypasses with the main channel in the ladder network as seen in Figure 5. If the point also has high shear gradient then the probability of thrombus nucleation increases (Fogelson, 2015) as shear gradient is shown to activate platelet adhesion.
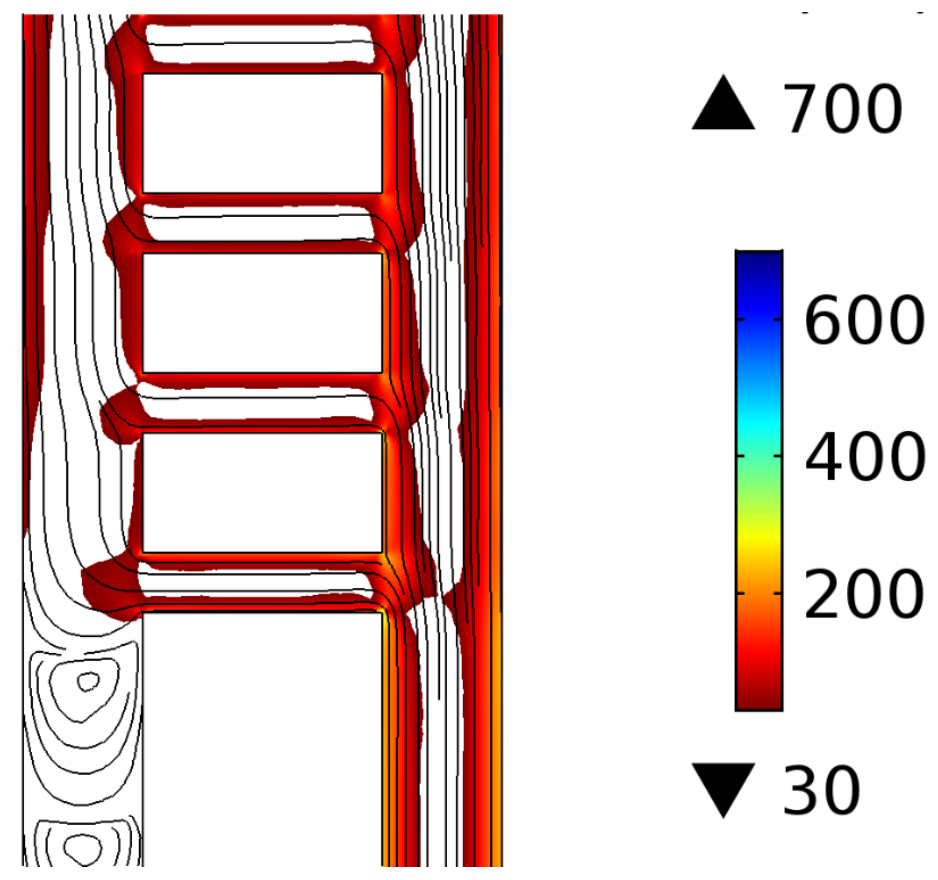

Figure 5 Shear rate and Streamlines plot of blood flow in ladder network-COMSOL

Nesbitt et al. have shown that platelets aggregate near regions of shear micro gradients. In addition, the aggregates can stabilize in presence of platelet agonists. For a thrombus to form the aggregates, need to stabilize at the point of nucleation. This means that we need to focus on mass transfer of thrombin, which can ensure stabilization of aggregates formed through shear micro gradients.

No-slip condition at the wall and the margination of platelets means that platelets will spend more time near the walls. Stagnation points occur when there is flow separation, especially 
when fluid hits an object and flows around it. In ladder network, we can expect stagnation points near the inlet walls of bypasses. In vasculature, stagnation points are located at the point of bifurcations as the vessels branch out. Figure 6 shows the velocity field with a stagnation point (black dotted circle) near the inlet of bypass 1 .

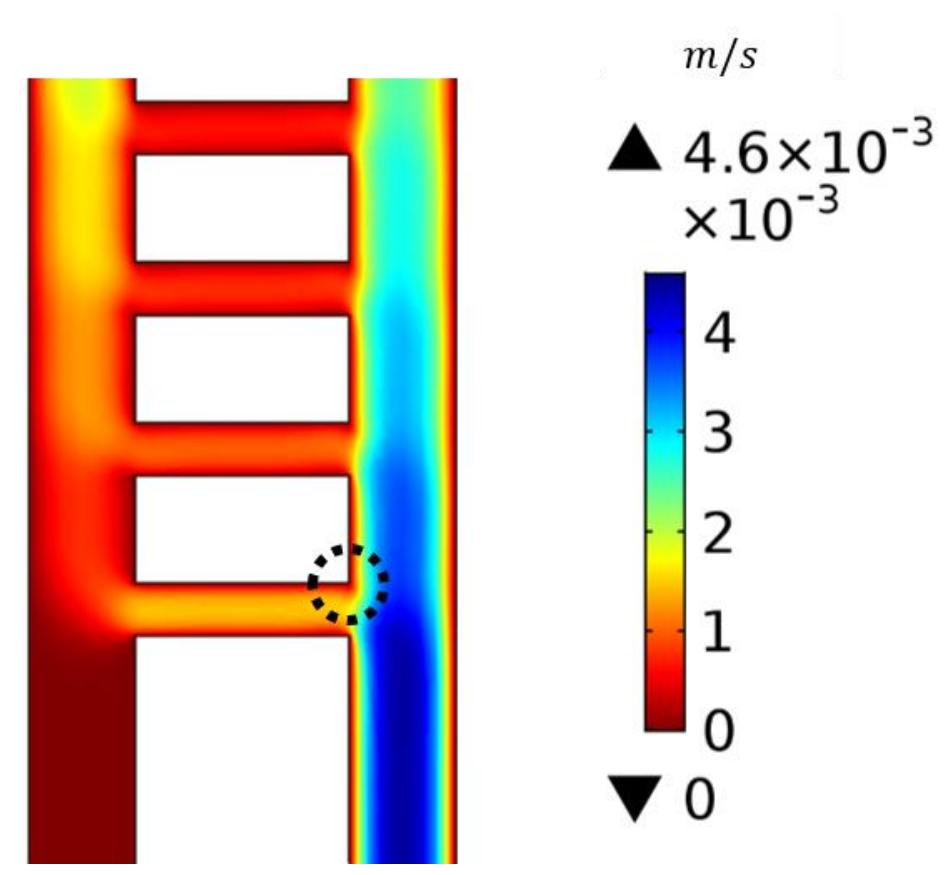

Figure 6 Velocity Field of blood in ladder network-COMSOL

Due to the difference in sizes of bypasses and main channels the shear rate changes abruptly near the intersection of inlet channel and first bypass. Due to margination at the inlet, $50 \%$ of the platelets entering the ladder network are marginated into the first bypass as can be seen in Figure 7.

The red circles are RBCs and the blue circles near the wall are platelets. This phenomenon is known as plasma skimming and is observed in vasculature that separates bloodstreams into RBC concentrated and diluted streams. 


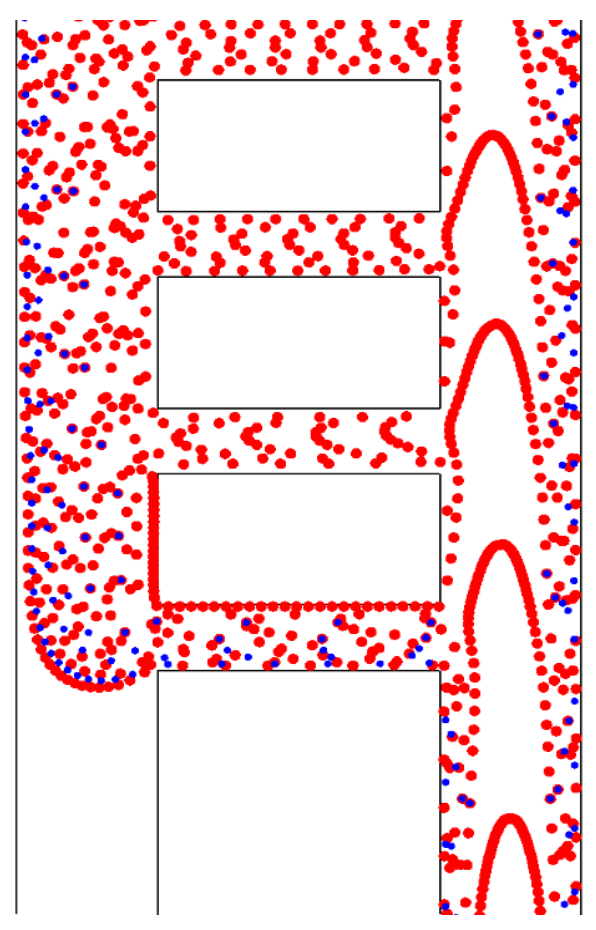

Figure 7 Trajectories of platelets and RBCs in ladder network

\subsection{Thrombin Convection}

When a thrombus forms at the inlet and outlet of bypass $-\mathrm{I}$, the activated platelets in the thrombus release thrombin to recruit more platelets to the aggregate. Thrombin is transported through diffusion and convection in the ladder network due to flow. It is assumed that thrombin is released from the surface of thrombus into the flow at a steady rate. A thrombus is modeled as a 2-D sphere with a constant thrombin surface concentration of $0.5 \mathrm{~mol} / \mathrm{m}^{3}$. These assumptions yield a qualitative result of thrombin transport into the ladder network which can be applied to determine secondary nucleation points in the ladder network. The continuity and steady state diffusion models are solved simultaneously to obtain total the thrombin flux in the geometry.

$$
\nabla \cdot\left(-D_{i} \nabla C_{i}\right)+u \cdot \nabla C_{i}=0
$$


$D_{i}-$ Diffusivity of thrombin $-10 \mu \mathrm{m}^{2} / \mathrm{s}$

$C_{i}-$ Thrombin Concentration $-0.5 \mathrm{~mol} / \mathrm{m}^{3}$ at the surface

The Peclet number, $P e$, in the ladder network is very high due to the low diffusivity of thrombin compared to the velocity of blood in the network. This results in convection being the dominant mechanism of thrombin transport.

$$
P e=\frac{\text { Rate of Advection }}{\text { Rate of Diffusion }}=\frac{L * u}{D_{\text {thrombin }}} \gg 1
$$

Figure 8 is the 2D surface plot of total thrombin flux along with streamlines in the ladder network. Red regions in the plot indicate maximum flux of thrombin. The flux is maximum in the direction of streamlines showing that convection dominates as the transport mechanism. In directions other than flow, the flux is almost zero, indicating poor diffusion of thrombin.

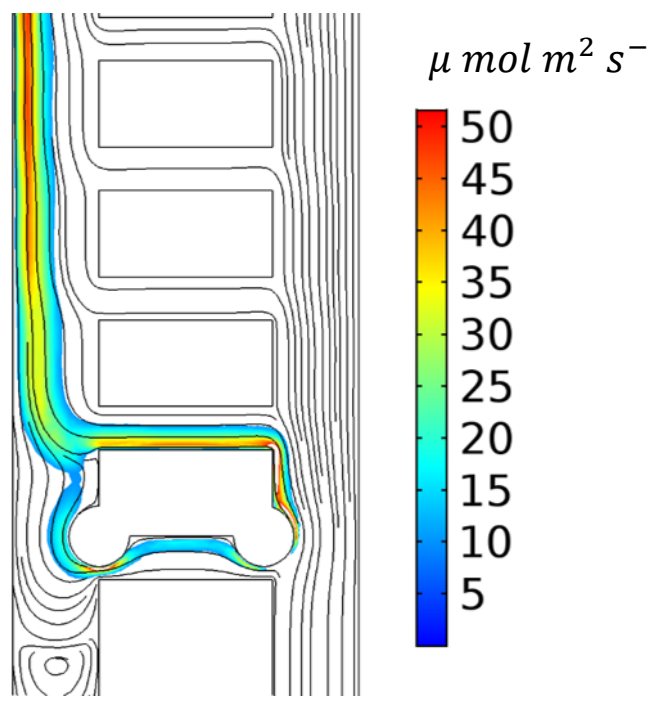

Figure $82 D$ Surface plot of Total thrombin flux in ladder network from COMSOL; Red regions in the plot signify higher thrombin flux, blue regions are the regions with lowest thrombin flux. Flux seen in the plot is higher along the streamlines showing the convection dominated thrombin transport 


\section{Thrombus predictions in ladder network}

A thrombus is formed when platelets are activated due to chemical triggers or mechanical triggers or both. Chemical triggers include activation due to thrombin and/or activation of platelets by tissue factor/collagen. Sub-endothelium contains collagen which comes in contact with the platelets when there is an injury. The platelets adhere to the collagen forming first layer of thrombus. Activated platelets secrete thrombin an enzyme that cleaves fibrinogen to form fibrin strands and activates nearby platelets to form thrombus. In the COMSOL simulations it is assumed that the entire device has collagen- a pro-coagulant coated on it. Due to flow, certain sites are expected to promote thrombus formation in the ladder network over the other sites. These sites with increased probability for thrombus formation can be identified through a set of metrics such as stagnation points, number of platelets, thrombin flux and shear gradient. In the following paragraphs, the metrics to identify thrombus nucleation sites are discussed.

\subsection{Metrics to Determine Thrombus Formation}

Prediction of thrombus nucleation points relied on estimating stagnation points, shear rate gradient and cell trajectories. Once the thrombus is nucleated, the thrombin transport rate was included along with the other parameters to determine secondary nucleation points. These four key parameters can be termed as metrics to locate the thrombus nucleation points in any geometry.

\subsubsection{Stagnation Points:}

The points at which the flow separation occurs are called stagnation points. In ladder network, the intersection of bypasses with main channel separated the flow from the main channel to the bypasses along with 'zero' velocity at those points (Figure 5). In a geometry, 
stagnation points are the first candidates for thrombus nucleation due to low velocity and flow separation.

\subsubsection{Shear gradient:}

Rapid change in shear rate from $700 \mathrm{~s}^{-1}$ in the main channel to around $300 \mathrm{~s}^{-1}$ in the bypasses can activate platelets and promote thrombus formation in the bypasses of ladder network (Figure 5). Stagnation points and shear rate gradient together predict the intersection of first bypass with the inlet and outlet channel as thrombus nucleation sites.

\subsubsection{Platelet Distribution:}

When the platelets enter the ladder network they are marginated with $50 \%$ of the platelets separating into the first bypass. The rest of the platelets get divided into other bypasses, which means when there are no clots in the device bypass 1 has the highest concentration of platelets. Estimating the distribution of platelets in the geometry coupled with stagnation points and shear rate gradient indicated bypass 1 to be the first bypass with thrombus. As the thrombus grows in bypass 1 , our model predicted that platelets move to bypass 2 due to decreased flow through bypass 1 . This leads to increased probability of thrombus formation near the entrance and exit of bypass 2 .

\subsubsection{Thrombin convection:}

Nucleation of thrombus due to stagnation points, shear gradient and higher platelet concentration serves as a thrombin source. We assumed the thrombi to be a constant concentration source of thrombin. Thrombin in bloodstream can activate platelets to release pro-coagulant chemicals thereby promoting thrombus formation. Our model predicted that 
thrombin is transported mainly through convection throughout the ladder network. Estimating the molar flow rate of thrombin lead to prediction of thrombus formation in outlet channel.

Overall, using these metrics of stagnation point, shear gradient, platelet concentration and thrombin molar flow rate the sites with high probability of thrombus formation in ladder network were predicted. Finite element simulations like these can be used to estimate the thrombogenic sites in any geometry. Figure 9 illustrates one way of applying these parameters in a systematic way to forecast thrombus nucleation sites.

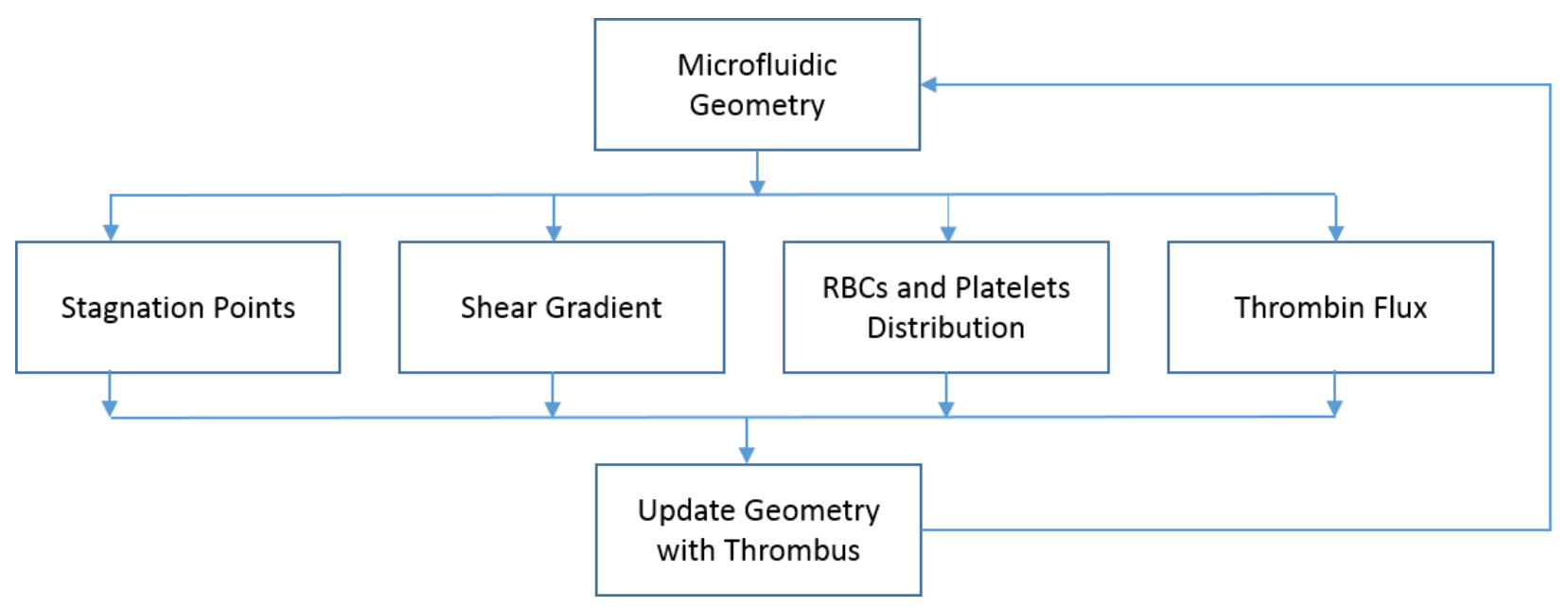

Figure 9 Modeling approach to prediction of thrombus formation patterns 


\subsection{Dynamic Thrombus formation}

Metrics discussed in the previous section were used to locate thrombus nucleation points in the ladder network. Once identified, a thrombus is assumed to grow in the spot in three stages of 5\%, $50 \%$ and $100 \%$ occlusion. At each stage of thrombus growth, the COMSOL geometry was modified and simulated to study the hemodynamic changes associated with the evolving geometry. The simulations were carried out for six cases of thrombus growth from bypass I to bypass V. Each case was assumed to last for 5 minutes, making it a total of 30 minutes of thrombus growth in ladder network.

Case 'zero' or 'null case' is the case of the ladder network with no clots. The metrics obtained through simulation of 'null case' lead to the intersection of first bypass with the main channel and the outlet channel as the nucleation points for clots shown with black circles in Figure 10 (a). In case 1, two thrombi of 5\% occlusion were generated in bypass I and the simulations were repeated with this updated geometry, Figure 10 (b), to generate shear rate, velocity, streamlines and thrombin flux based on the conditions under section 3.1. In the next case, case 2, shown in Fig 13 $\mathrm{b}$, the clots in bypass I were increased in size to $50 \%$ occlusion of bypass I and two new clots were incorporated into the geometry at bypass II of size 5\% occlusion. In case 3, clots in bypass I were moved to $100 \%$ occlusion, completely blocking the flow through bypass I and clots in bypass II reached 50\% occlusion while two new clots of 5\% occlusion are created in bypass III. Each of these cases were assumed to last for 5 minutes within which a steady state is assumed to be established in the geometry.

Every time the thrombus in $\mathrm{n}^{\text {th }}$ bypass reaches $50 \%$ occlusion, the $(\mathrm{n}+1)^{\text {th }}$ bypass will have a $5 \%$ clot as shown in Figure 10. Focus of these cases was to understand the change in shear rate, number 
of platelets through bypasses and thrombin transport in the ladder network as the ladder geometry evolved due to formation of thrombus.

(a)

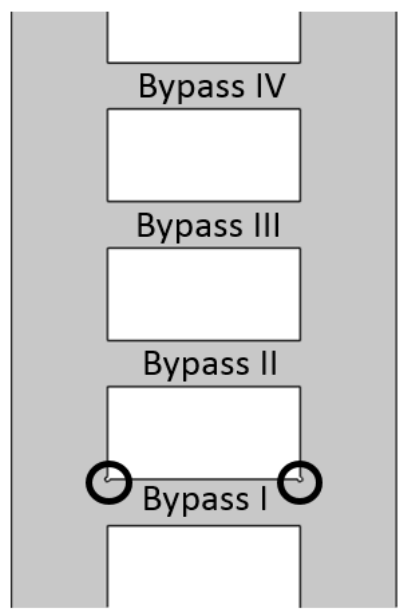

(d)

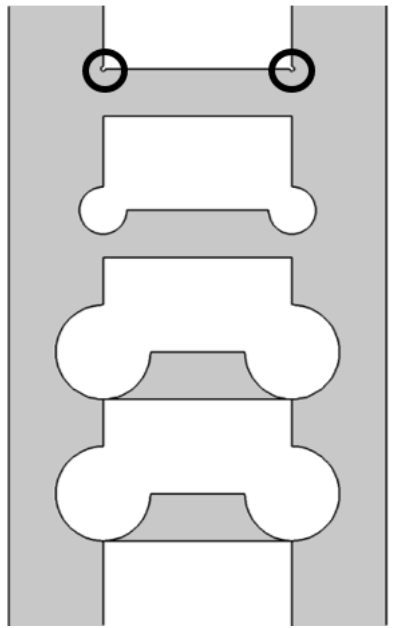

(b)

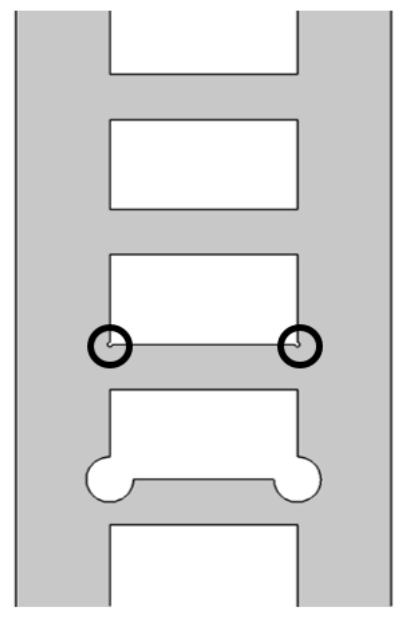

(e)

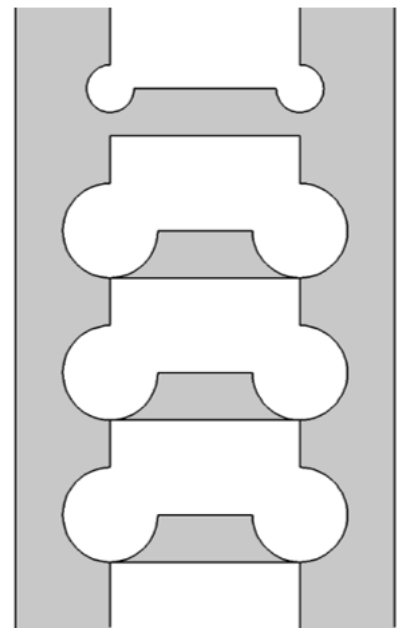

(c)

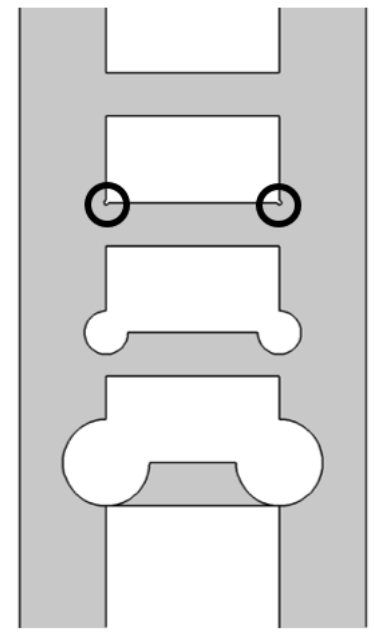

(f)

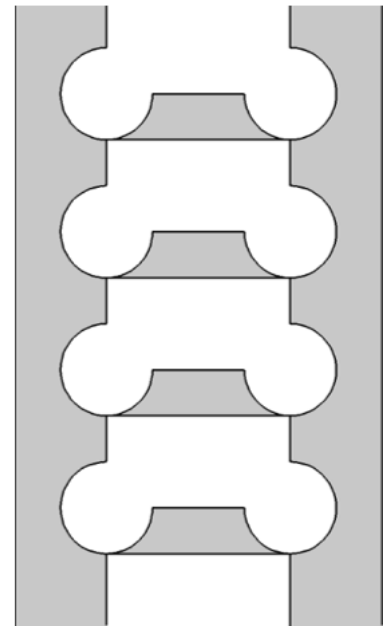

Figure 10 Thrombus evolution dynamics in the ladder network. a) Case 1 bypass I 5\% occlusion; b) Bypass I 50\% and bypass II 5\% occlusion; c) Bypass I 100\%, bypass II 50\% and bypass III 5\% occlusion; d) Bypass I, II 100\%, bypass III 50\% and bypass IV 5\% occlusion; e) Bypass I,II, III, 100\%, bypass IV 50\% and bypass V 5\% occlusion; f) Bypass I,II,III and IV 100\% and bypass V 50\% occlusion 


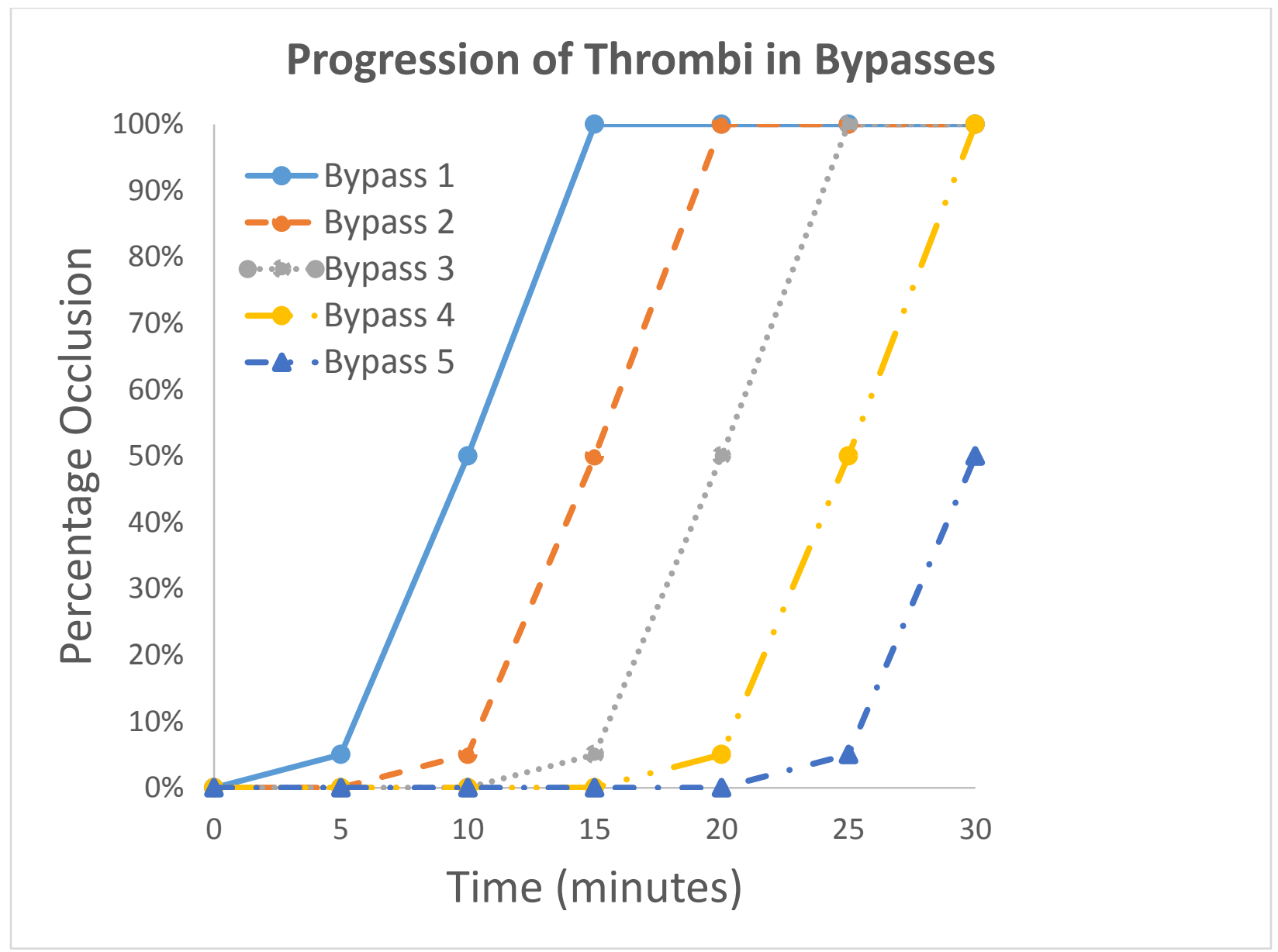

Figure 11 Occlusion Pattern simulated in COMSOL for bypass 1 to 5 . Each set of thrombi in ( $n+1)$ th bypass move from $0 \%$ occlusion to $100 \%$ occlusion in 15 minutes after the thrombi in the nth bypass.

\subsubsection{Shear rate evolution:}

A Cut line method was employed in COMSOL to determine the shear rate in each bypass for every case of dynamic thrombus formation. In this method, COMSOL returns the values of shear rate along a cut line drawn in the model. These values then can be imported to Excel, where they can be processed to sort the values at interested $(\mathrm{x}, \mathrm{y})$ locations of the model. Figure $12(\mathrm{a}-\mathrm{c})$ shows case 2, where the cut lines for each measurement are represented as arrows. The values reported in the plot are the values derived from the data obtained along these cut lines in COMSOL. 
(a)

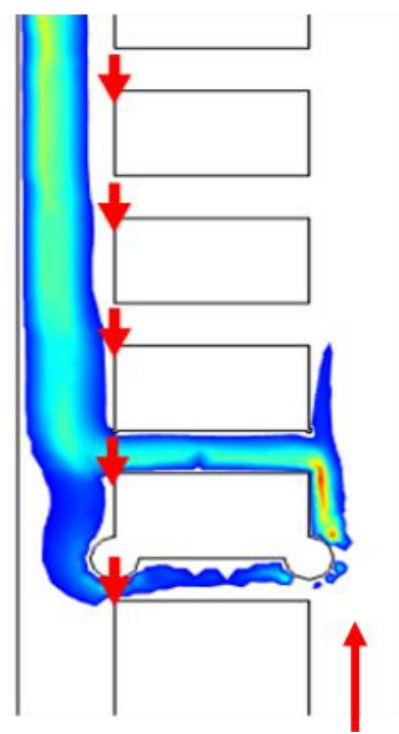

(b)

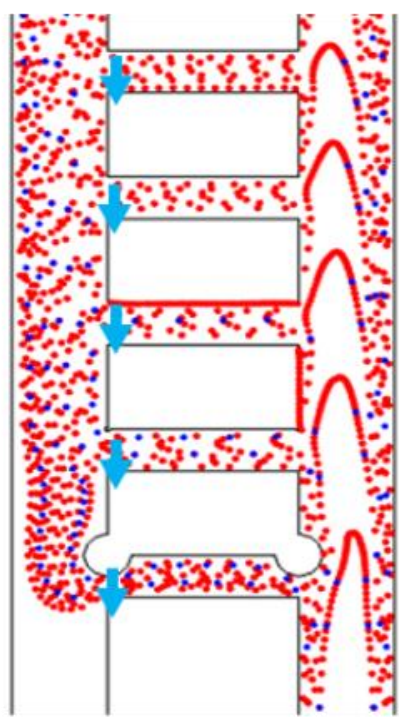

(c)

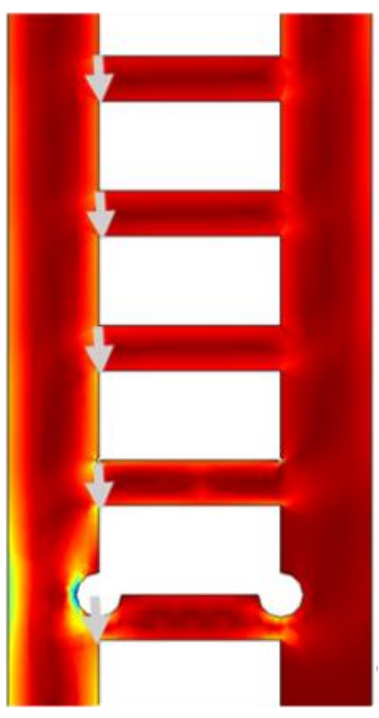

Figure 12 Cut Line method for measuring (a) Total thrombin flux, (b) Percentage of platelets in bypasses and (c) Shear rate

The maximum shear rates measured after the simulation of each case in all the bypasses indicate that shear rates show a non-linear behavior with thrombus growth. At 5 minutes, when the bypass I reaches 5\% occlusion, the shear rate for bypass I is maximum (Figure 13). For bypass II, at 10 minutes when there is $5 \%$ occlusion the shear rate is maximum later going to ' 0 ' at $100 \%$ occlusion because of no flow. All the bypasses had the maximum shear rate at 5\% occlusion. In this network, maximum shear rate on the thrombus surface was observed for $5 \%$ occlusion for thrombi in all bypasses. This feature is unique to every geometry and boundary condition as verified through two channel experiments reported in literature (Thomas V. Colace, 2012). 


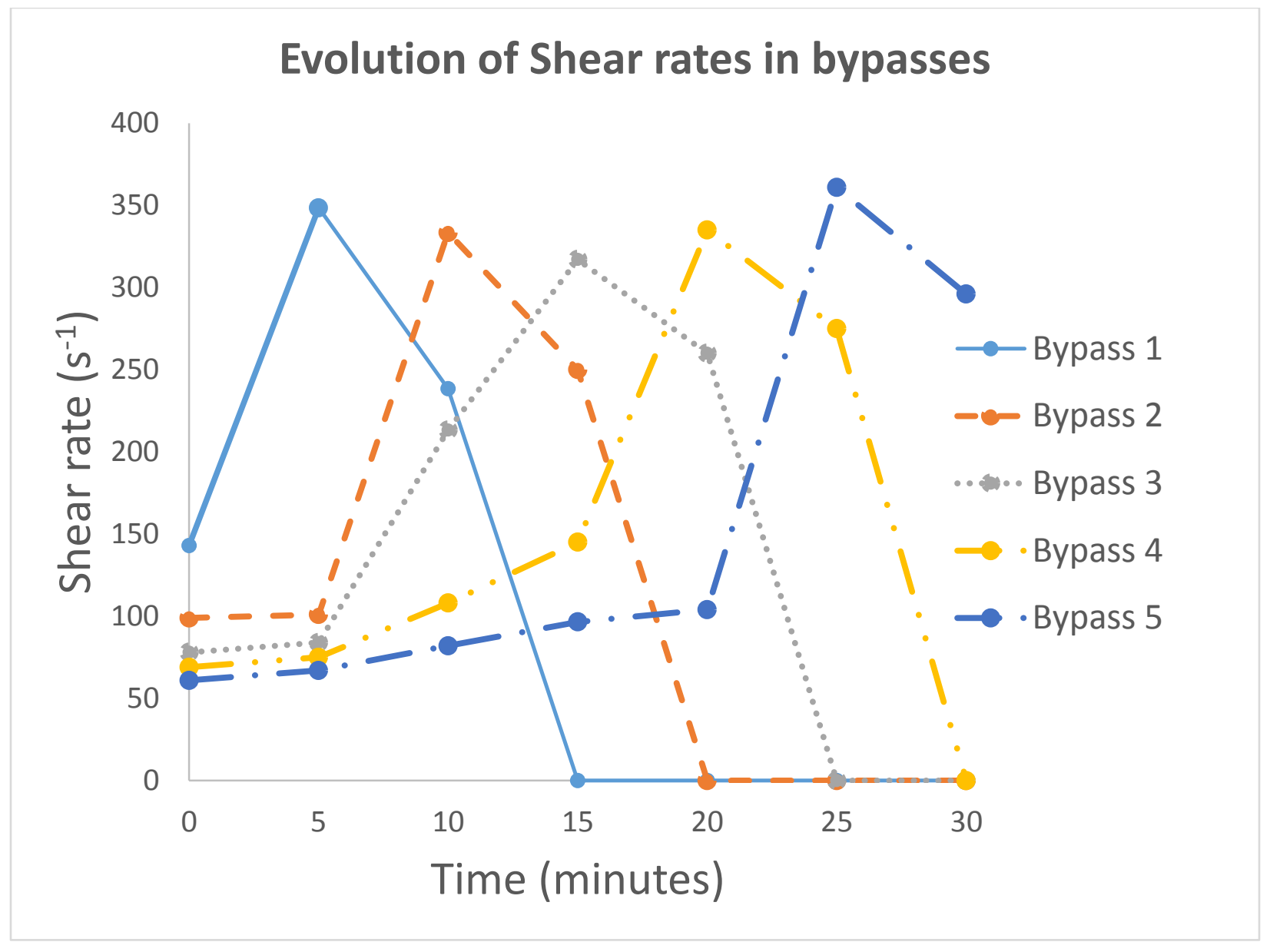

Figure 13 Maximum shear rate in bypasses at all the stages of thrombus progression.

\subsubsection{Cell distribution:}

The effect of thrombus growth on particle trajectories is accounted through measuring the percentages of platelet in each bypass for all the cases. Particle counter at the exit of each bypass was used in COMSOL to determine the number of platelets passing through each bypass. In the 'null case', $50 \%$ of the platelets moved into the bypass I. As we initiated a thrombus in bypass I in after 5 minutes (case 1), this percentage comes down to $30 \%$ later decreasing to $0 \%$ when the bypass is completely occluded. As the thrombus grows in $\mathrm{n}^{\text {th }}$ bypass the platelets are diverted to $(n+1)^{\text {th }}$ bypass increasing the probability of thrombus formation in $(n+1)^{\text {th }}$ bypass. The formation 
of thrombus depends highly on the availability of platelets, a stagnation point without platelets around it cannot have a thrombus.

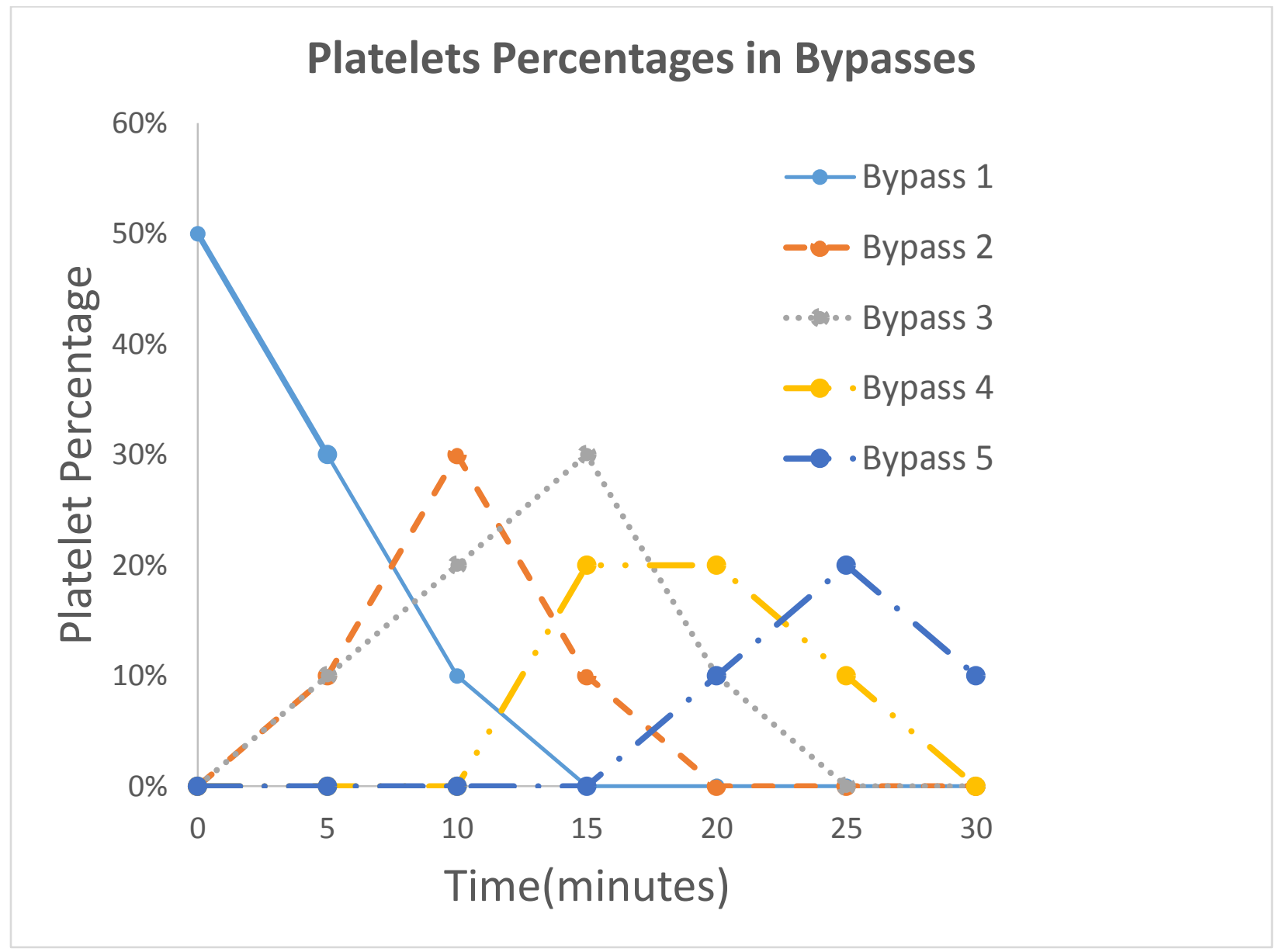

Figure 14 Effect of thrombus formation on the number of platelets in bypasses.

\subsubsection{Total thrombin flux:}

A cut line was drawn at the exit of each bypass to measure the total thrombin flux at the outlet of each bypass. As the thrombus in bypass 1 went to $50 \%$ occlusion after 10 minutes, the molar flow rate of thrombin from bypass 1 reaches a maximum. However, at the end of 15 minutes, even 
though the thrombi have grown larger in bypass 1 , most of the thrombus surface area, source of thrombin, is blocked inside the bypass through which flow is obstructed. This leads to decrease in total flux of thrombin from bypass 1 .

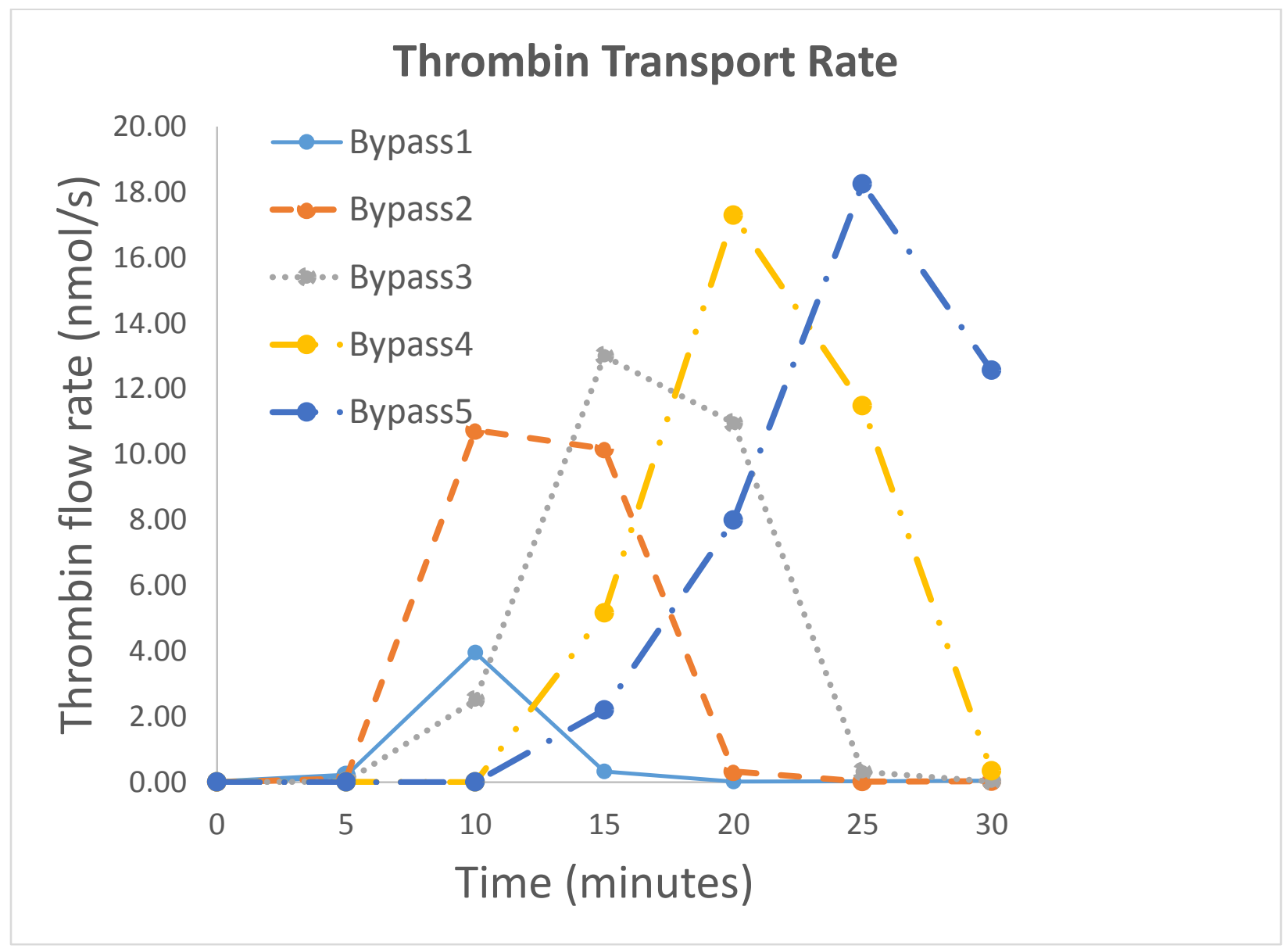

Figure 15 Rate of thrombin transport from each bypass. Thrombin transport increases with thrombus growth till $50 \%$ occlusion. 


\section{Microfluidic ladder network experiments}

The validation of nucleation points prediction from the COMSOL model against the experimental data from a microfluidic ladder device is discussed in this chapter. The fabrication

of microfluidic device followed by the description of experimental data and comparison of simulation results with the experimental data are discussed under the following titles.

The microfluidic device for the experiment was fabricated at the clean room facility of Shared Research Facilities (SRF) at West Virginia University. Experimental data was gathered from Dr. Owen McCarty's lab at the Oregon Health and Science University, Biomedical engineering department.

\subsection{Fabrication of microfluidic ladder network}

Microfluidic ladder network was fabricated using photolithography technique. The technique involves four important steps.

1. Designing the device in software such as AutoCAD, Adobe Illustrator.

2. Printing the designs onto a transparency mask

3. Using the transparency mask to fabricate a master with the device design

4. Molding of microfluidic device with polydimethylsiloxane (PDMS)

Ladder network was designed using AutoCAD and an external printing service was employed to print the design on a transparent film like mask. Silicon wafers are used as the masters in the photolithography technique for their excellent resolution and pattern quality. Photolithography 
was carried out in the clean room facilities of Shared Research Facilities (SRF) of West Virginia University.

Silicon wafers are cleaned with acetone to remove all contaminants and then rinsed with isopropyl alcohol (IPA). Clean wafers are dehydrated in an hot plate and then cooled down in room temperature. The silicon wafers are then spin coated with SU-8 gel, a negative photoresist used for fabrication of devices in the range of 10 to 100 microns thickness. Once coated, the wafer is then soft baked on a hot plate and then cooled down to room temperature. The coated silicon wafer was aligned under the photo mask with the ladder design and treated with Ultra Violet (UV) radiation.

The UV light etches the design on the photomask on to the silicon wafer with SU-8 coating. The UV exposed silicon wafers are baked again in a hot plate for about 6 minutes at $65^{\circ} \mathrm{C}$ and then cooled down to room temperature. Silicon wafer with the design etched on it is immersed into the developer solution for a time suggested by the photoresist manufacturer. This developed wafer is called the silicon master which can be used to mold microfluidic ladder devices. Features of ladder network design on the master, were then verified using a microscope.

The microfluidic ladder was molded using a 10:1 ratio (w/w) of Sylgard 184 polydimethylsiloxane (PDMS) polymer to curing agent from the silicon master. A BD-20AC Corona Treater (ElectroTechnic products, Inc) was used to plasma bond PDMS molds onto microscopy slides. Figure 16 (a) shows the microfluidic ladder network as visualized by a differential interference contrast microscopy (DIC). Figure 16 (b) is the experimental set up of the microfluidic device fixed with blood pumped through it. The ladder device was coated with collagen and recalcified whole human blood was pumped through it at a flow rate of $2 \mu \mathrm{L} / \mathrm{min}$. The results of the experiment were reported as images recorded using Zeiss Axio Imager 2 microscope. A more detailed description 
of the experimental methods can be found in the journal article (Zilberman, Sylman, Lakshmanan, J.T.McCarty, \& Maddala, 2017).

(a)

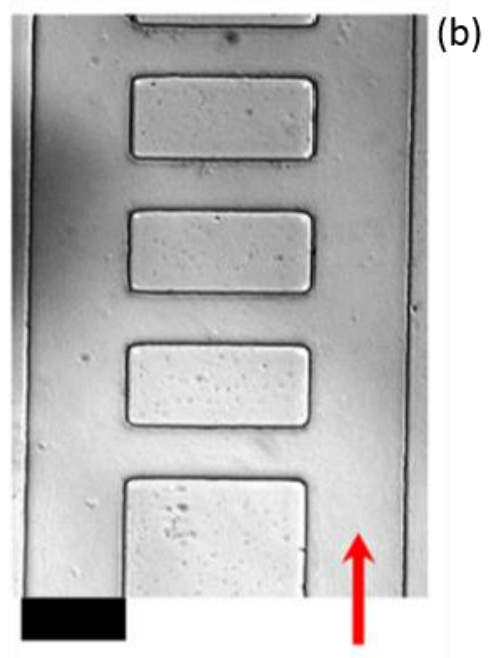

(b)

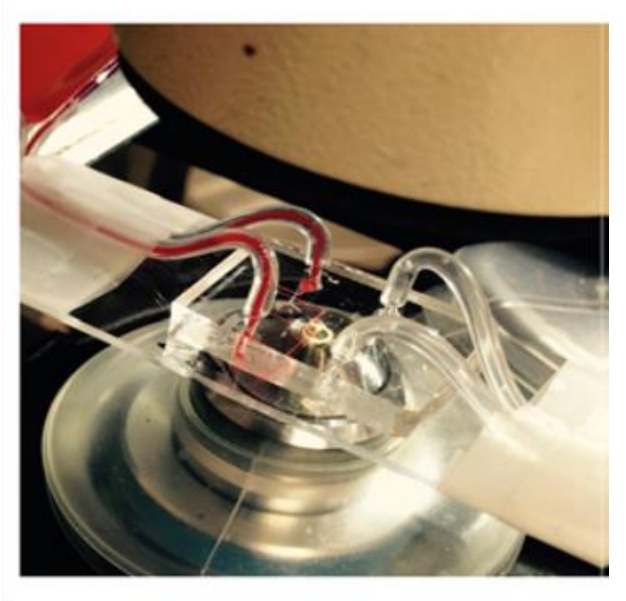

Figure 16 (a) Microfluidic ladder network as seen under a Differential Interference Contrast (DIC) microscope (b) Experimental setup of the ladder network with the blood pumped through the device

\subsection{Thrombus formation in Microfluidic Ladder Network}

Blood perfusion expriments on the microfluidic ladder network looked for thrombus in the ladder network. Figure 17 is the image of microfluidic ladder network after 30 minutes of blood perfusion. Experimental image shows that clots formed at the intersection of all bypasses and main channels, near the inlet and the outlet. The red dashed circles in Figure 17 indicate the initial clots that were formed in the ladder network, these clots are predicted through platelet trajectories, shear gradient and stagnation points from the modeling. Clots in the main channel (yellow solid circle in Figure 17) are explained through activation of platelets through thrombin convection from the clots in bypass I. 


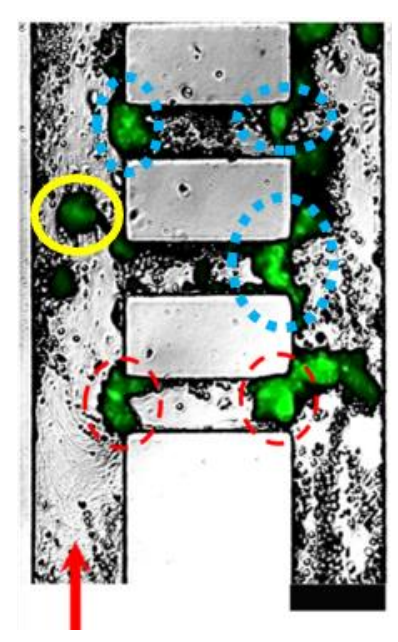

Figure 17 Experimental images of Thrombus in microfluidic Ladder network.

Red broken line circles indicate the clots predicted through stagnation points, shear gradient and cell distribution, blue dotted circles indicate secondary clots predicted through metrics and yellow solid circle is the clot predicted through thrombin convection

More platelets move into bypasses II, III and IV as clots grew in the preceding bypasses. Prediction from the dynamic thrombus simulations revealed the pattern behind increase in platelet numbers and the non-linear shear rate behavior in bypasses. Dynamic thrombus simulations and the metrics developed in the model, shear gradient, thrombin convection, platelet distribution and stagnation points describe the clots marked in dotted blue circles in Figure 17.

Overall the COMSOL model described the flow of blood, shear rates, distribution of cells- platelets and RBCs, and the transport rates of thrombin in the ladder network. Quantifiable metrics developed from the model were used to identify the thrombus nucleation sites in the ladder network. These metrics could be used as a preliminary markers to find the probable thrombus sites in any vascular network. However, these parameters could not describe the occlusion scenario of thrombus vis-à-vis location and architecture of the network. The next chapter describes our approach to identify the relation between geometrical parameters and the occlusion scenario of thrombus. 


\section{Thrombus stability}

A thrombus in a vessel can either disintegrate or stay intact. Disintegration of thrombus can happen in two ways, thrombolysis and embolization. Tissue plasminogen activator (t-PA) is released into the blood after the injury is healed to activate plasminogen to plasmin. Plasminogen is a protein in blood produced by liver. Plasmin cleaves the fibrin strands binding the platelet aggregate to dissolve the clot. This phenomenon is called thrombolysis or sometimes referred to as fibrinolysis (R.G.Macfarlane \& Rosemary Biggs, 1948). When a thrombus does not disintegrate, it breaks off by the shear forces around the thrombus and is termed as embolization. A thrombus that neither disintegrates nor embolizes continues to grow and occlude the vessel leading to dangerous consequences. The problem statement for thrombus stability is defined in a way that identifies the effect of geometry on clot occlusion.

\subsection{Problem Statement:}

(a)

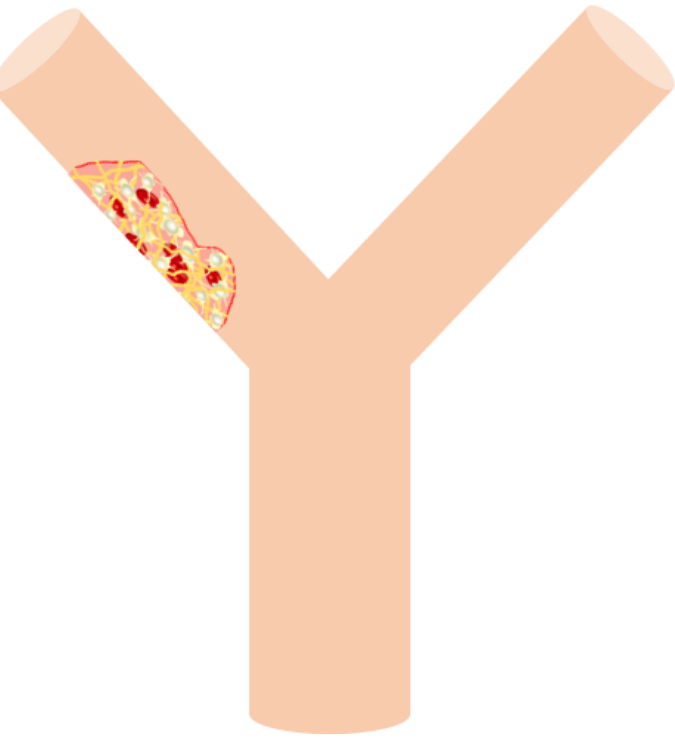

(b)

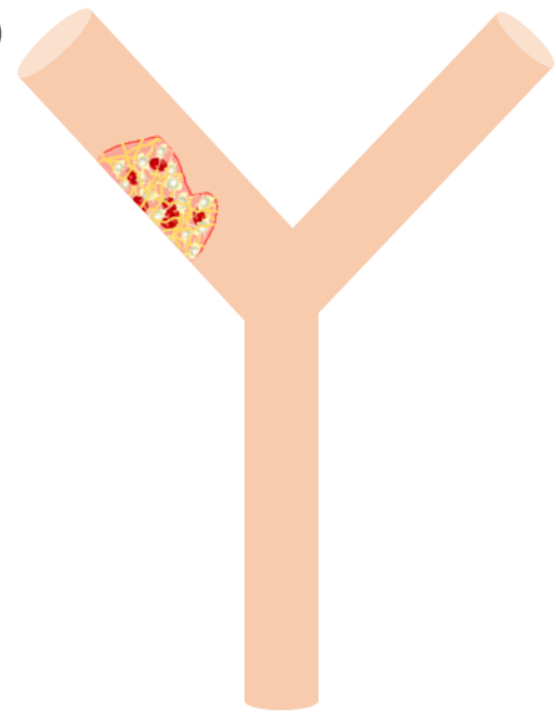

Figure 18 Thrombus Stability in different geometries- Which thrombus will occlude the vessel ? 
Figure 18 (a) and (b) are two bifurcations with different radii of branches, and parent vessel with a longer thrombus on Figure 18 (a) and a shorter thrombus on Figure 18 (b). The question is "Which of these thrombi, will grow to occlude the vessel and which will stay below complete occlusion? Is this dependent on the architecture of bifurcation?". The answers to these questions come from understanding the stability of thrombus in a straight channel and then integrating these results into a bifurcation model.

The current models in the literature for thrombus growth rate are investigated followed by the study of evolution of shear rates around a thrombus in a straight channel. Using the models available to determine the occlusive stability of thrombus in straight channel, a model to identify stability of thrombus in a bifurcation is developed. Finally the implications and limitations of the model are discussed.

\subsection{Models for Growth Rate of Thrombus}

Experimental observations from literature revealed that a thrombus consists of a shell and core (Stalker, E.A.Traxler, \& L.F.Brass, 2013). The core part of thrombus contains firmly adhered platelets, while the shell of the thrombus has platelets getting attached due to activation and detached due to shear forces continuously. The net growth rate of thrombus depends on the attachment of platelets onto the thrombus shell (Falati, 2002). The existing models on the growth rate of thrombus under flow (Tokarev, Butylin, \& Ataullakhanov.F.I, 2011) describe the non-linear dependence of platelet aggregation on wall shear rate.

Belyaev's reduced model (Belyaev, Panteleev, \& Ataullakhanov, 2015) considers the growth of thrombus as the difference between platelet attachment and detachment rate at the shell of thrombus. Thrombus growth involves activation of platelets, cleavage of fibrinogen, and 
attachment of platelets. Modeling the myriad biological reactions will take the focus away from understanding the physics of thrombosis. The non-hydrodynamical phenomena involved in determining the growth rate of thrombus are accounted for in Belyaev's model through the parameters $\varphi$ and $\omega_{d}$.

$$
k_{\text {eff }}=\text { Platelet influx }- \text { Platelet Outflux }=\varphi \dot{\gamma}^{1-\beta}-\omega_{d} \dot{\gamma}
$$

$k_{\text {eff }}-$ Effective growth rate of thrombus, in $\mathrm{m} / \mathrm{s}$

$\varphi-$ Accounts for hematocrit and near wall concentration of platelets, $\mathrm{m} \mathrm{s}^{-0.2}$

$\omega_{d}$-Thrombus erosion constant determined through experimental data, $\mathrm{m}$

$\beta$ - Shape parameter, depends on the sizes of platelets and $\mathrm{RBCs}, 0.2$ for human blood cells

The $k_{\text {eff }}$ in the model has units of $\mathrm{m} \mathrm{s}^{-1}$ and describes the effective growth rate of thrombus.

This model captures the non-linear dependence of thrombus growth rate on shear rate. The rate of detachment of platelets increases proportional to shear rate while rate of attachment of platelets increases linearly with shear rate at lower shear rates and decreases at higher shear rates. The growth rate of thrombus defined through Belyaev model can be visualized as an equilibrium process. A non-occlusive thrombus reaches the equilibrium of zero growth rate before $100 \%$ occlusion, where it seals the injury without dangerous consequence of blocking blood supply. 


\subsection{Critical Shear Rate}

The shear rate at which the effective growth rate of a thrombus becomes zero is defined as the critical shear rate. At critical shear rate, rate of thrombus erosion equals the rate of thrombus growth, i.e. $k_{e f f}=0$. Critical shear rate is given by the ratio of the two parameters $\varphi$ and $\omega_{d}$ raised to the power of $1 / \beta$.

$$
\dot{\gamma_{c r}}=\left(\frac{\varphi}{\omega_{d}}\right)^{\frac{1}{\beta}}
$$

Belyaev et al. used the rat in vivo experimental data of thrombus growth (Sato \& Ohshima, 1986) to estimate the critical shear rate for rat vasculature as $3400 \mathrm{~s}^{-1}$. Analytical estimation of critical shear rate for human microvasculature becomes difficult as the parameters $\varphi$ and $\omega_{d}$ require experimental data for estimation.

Experimental data from microfluidic ladder network (Figure 17) also show that geometry can not only control the nucleation but also regulate the extent of growth of thrombus. The thrombi growing in the bypass occluded the bypass while the thrombus in main channel stopped growing at partial occlusion. Shear rates in the main channel are much higher than the shear rates in bypasses due to constant inlet flow rate. Geometry's relation with thrombus stability can be established through shear rates. Critical shear rate can help us establish the stability of thrombus in different geometries. Thrombi form in irregular shapes in different areas of vascular system. Critical shear rate identification in arteries and veins would be difficult owing to presence of higher Reynold's number flows. However, in 
microvasculature $(100-1000 \mu \mathrm{m})$ the $R e<1$ and the assumption of Newtonian flow is more valid. The following sections describe the dynamics of shear rate on the surface of thrombus growing in microvasculature. It is assumed that the length of thrombus remains constant and the Hagen Poiseuille equation can describe the flow rate dynamics. In-vivo experiments show that assumption of constant thrombus length is adequate as long as the concentration of activators in the injury site is moderate.

\subsection{Shear rate analysis in a Straight Channel}

A growing thrombus in a blood vessel increases the resistance of the vessel. This straight channel can be visualized as three hydraulic resistances in series, $R_{1}, R_{2}$ and $R_{3}$. The resistance $\mathrm{R}_{2}$ corresponds to thrombus, Figure 19 . As $\mathrm{R}_{2}$ increases the flow rate through the channel changes thereby changing the shear rate on the thrombus surface. Thrombus is irregular in shape, however, assumption of a rectangular thrombus growing under laminar flow permits the application of Hagen Poiseuille equation to estimate the shear rate around the thrombus analytically. Laminar flow is a valid assumption for microvasculature where vessels range in size from $50-1000 \mu \mathrm{m}$.

$$
\begin{gathered}
\left.Q=\frac{\Delta P}{R} \text { (Hagen Poiseuille law }\right) \\
\left.\dot{\gamma}=\frac{8 v}{D}=\frac{32 Q}{\pi D^{3}} \text { (Laminar flow }\right) \\
Q=\frac{\Delta P}{R_{1}+R_{2}+R_{3}} \\
R_{i}=\frac{128 \mu l_{i}}{\pi D_{i}{ }^{4}}
\end{gathered}
$$


The hydraulic resistances $R_{1}$ and $R_{3}$ remain unchanged while the resistance $R_{2}$ increases as the thrombus grows, through decreasing $\mathrm{D}_{2}$. The length of thrombus ' 12 ' is fixed constant throughout the process. The total flow rate through the channel at any instant is given by the following equation.

$$
Q=\frac{\Delta P}{\frac{128 \mu l_{1}}{\pi D_{1}^{4}}+\frac{128 \mu l_{2}}{\pi D_{2}^{4}}+\frac{128 \mu l_{3}}{\pi D_{3}^{4}}}
$$

The shear rate on the surface of thrombus is estimated from the below equation with the flow rate estimated from the above equation.

$$
\dot{\gamma}_{2}=\frac{32 Q}{\pi D_{2}{ }^{3}}-\text { Shear rate on thrombus surface }
$$
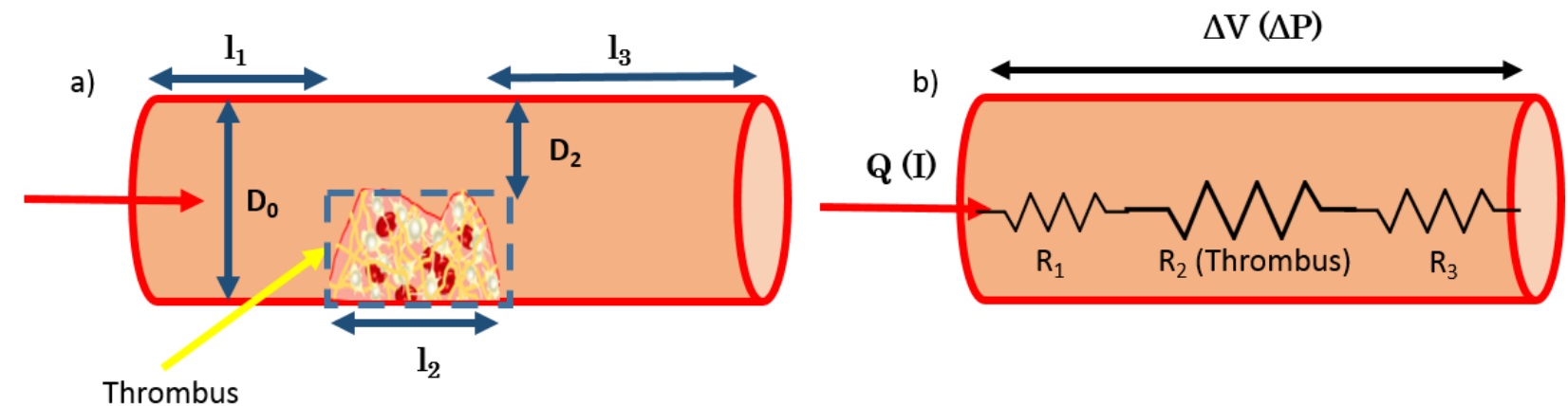

Figure 19 Thrombus growth in a straight channel, $D_{0}$ is the initial diameter of the channel, $D_{2}$ is the open diameter at any instant during thrombus growth, $I_{2}$ is the length of thrombus. Pressure Drop is analgous to Potential difference and flow rate is analgous to current in an electrical circuit

$\mathrm{L}$ and $\mathrm{h}$ denote the length and height of the thrombus. Length of thrombus can be assumed constant based on experimental evidence that shows for weaker injuries the thrombus length does not change during growth (Okorie, 2008). We assume blood is a Newtonian fluid and 
the flow is laminar. Here the flow of blood is analogous to the flow of current and the pressure drop across the channel is analogous to the potential difference in an electrical circuit.

The mathematical model was examined by increasing $\mathrm{R}_{2}$ to understand the shear rate dynamics in the region of thrombus. The evolution of shear rate depends on the boundary condition employed in the vessel. The shear rate dynamics under two different boundary conditions, constant flow rate and constant pressure drop has been analyzed in the following sections.

\subsubsection{Constant Flow Rate}

' $D_{0}$ ' is the initial diameter of the vessel and ' $\mathrm{D}_{2}$ ' is the unblocked diameter of the thrombus portion of vessel. We define percentage occlusion from the ratio of these two diameters.

$$
\text { Percentage Occlusion }(x)=\left(1-\frac{D_{2}}{D_{0}}\right) X 100
$$

Initially when thrombus growth is zero, $\mathrm{D}_{2}=\mathrm{D}_{0}$ and $\mathrm{X}$ is zero. Finally when the thrombus fully blocks the vessel, $\mathrm{D}_{2}=0$ and $\mathrm{x}=100 \%$.

Shear rate on the thrombus under constant flow rate increases monotonically eventually reaching infinity at $100 \%$ occlusion as seen in Fig 9. Constant flow rate experiments with straight channel have shown similar results (Thomas V. Colace, 2012). This shows that thrombi growing under constant flow would eventually break off when the shear stress reaches very high values. Colace's experiments on straight channel showed this yielding stress to be $29,000 \mathrm{~s}^{-1}$ for a clot composed primarily of fibrin. If the initial shear rate in the vessel is less 
than the critical shear rate, then during growth the shear rate would reach critical shear rate. Monotonic increase in shear rate under constant flow rate is due to decreasing diameter in the denominator in equation (20). A constant flow rate leads to increasing values of shear rate as D decreases due to thrombus growth.

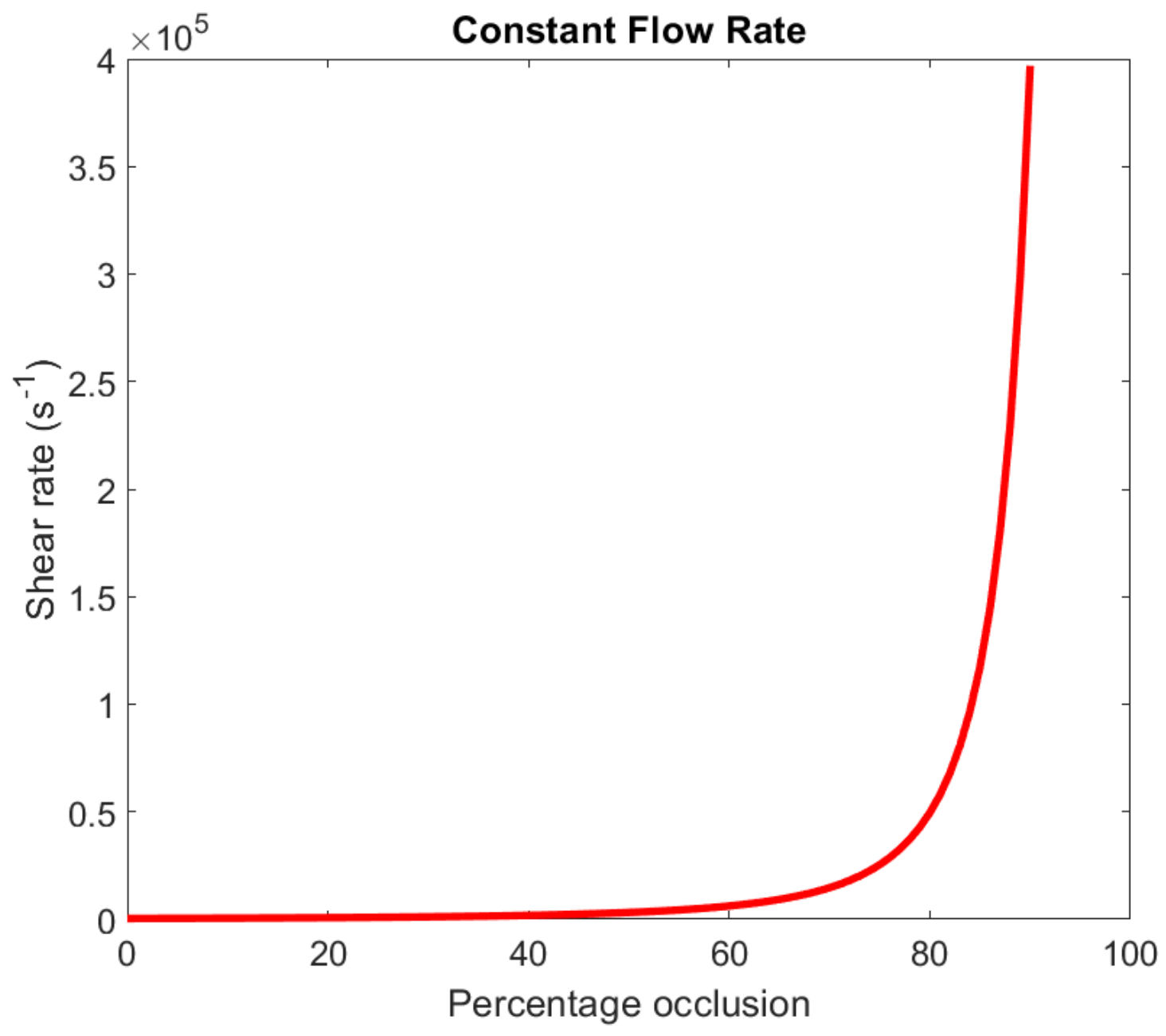

Figure 20 Shear rate on the thrombus in a straight channel under constant flow rate

\subsubsection{Constant Pressure Drop}

When the pressure drop across the channel is maintained constant, equation (19) was solved for flow rate for each step of thrombus growth and the shear rate was determined using 
equation (20). The decrease in flow rate is proportional to the increase in resistance gives rise to a non-linear behavior of shear rate as shown in Figure 20.

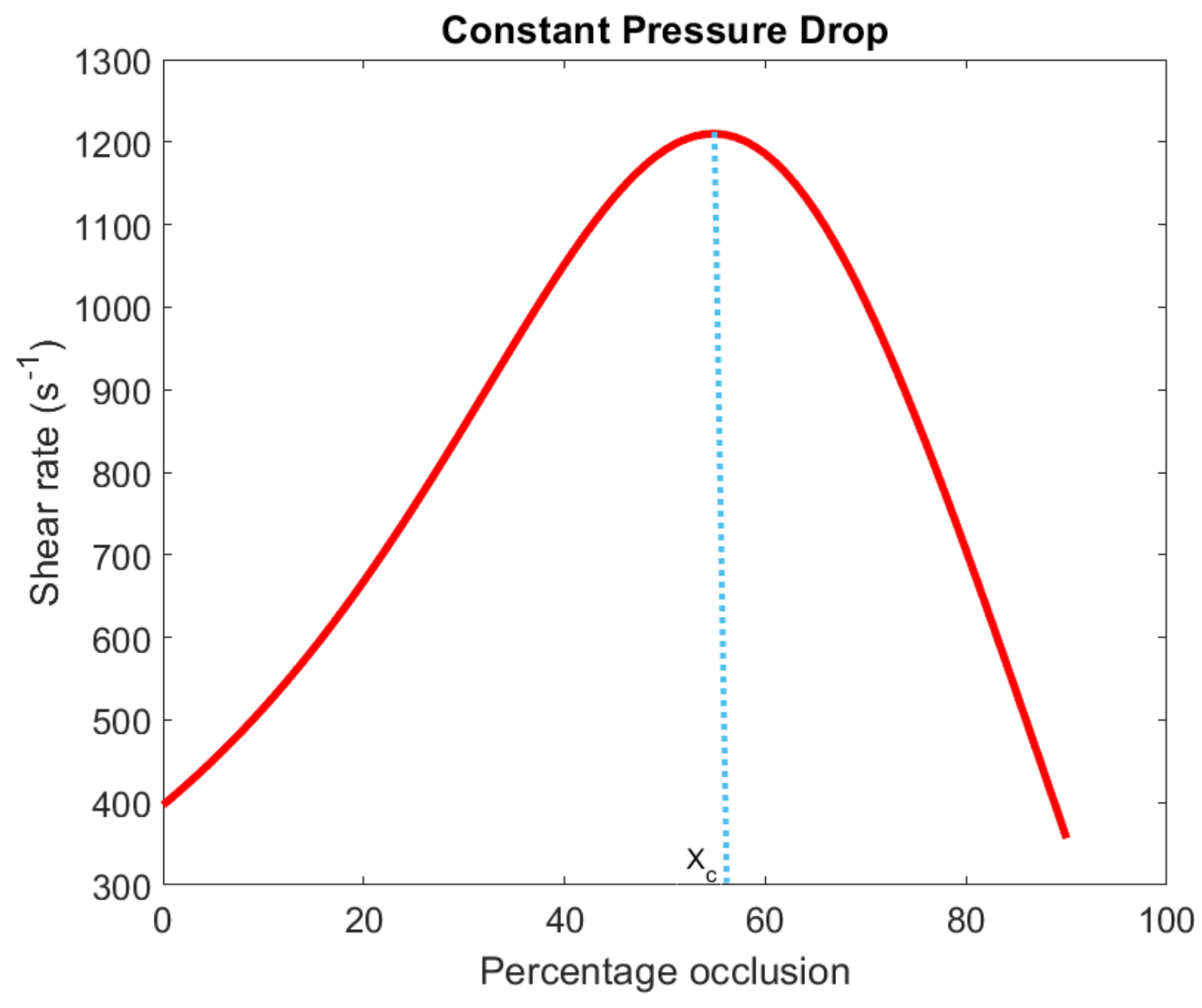

Figure 21 Shear rate on the thrombus in a straight channel under constant pressure drop

These results correspond to the shear dynamics under constant pressure drop reported in literature (Thomas V. Colace, 2012). The shear rate in the region around the thrombus grows initially with occlusion percentage with a maximum occurring at around $60 \%$ occlusion before it decreases to zero at $100 \%$ occlusion. The critical occlusion at which maximum shear rate occurs depends on the length of thrombus itself (with everything else being constant), longer thrombus will have $\mathrm{X}_{\mathrm{c}}$ close to $0 \%$ and shorter thrombi will have $\mathrm{X}_{\mathrm{c}}$ close to $100 \%$. The effect of length of thrombus on the behavior of shear rate can be seen in Figure 21. As the thrombus 
gets longer, the non-monotonic behavior of shear rate changes to monotonically decreasing behavior. This change in behavior is due to the fact that longer thrombus shifts the maximum closer towards $\mathrm{X}_{\mathrm{c}}=0$ and in our case when length of thrombus was $70 \%$ the length of the entire blood vessel, the maximum shear rate occurs at $X_{c}=0$. This implies that the initial shear rate is the maximum shear rate in this case.

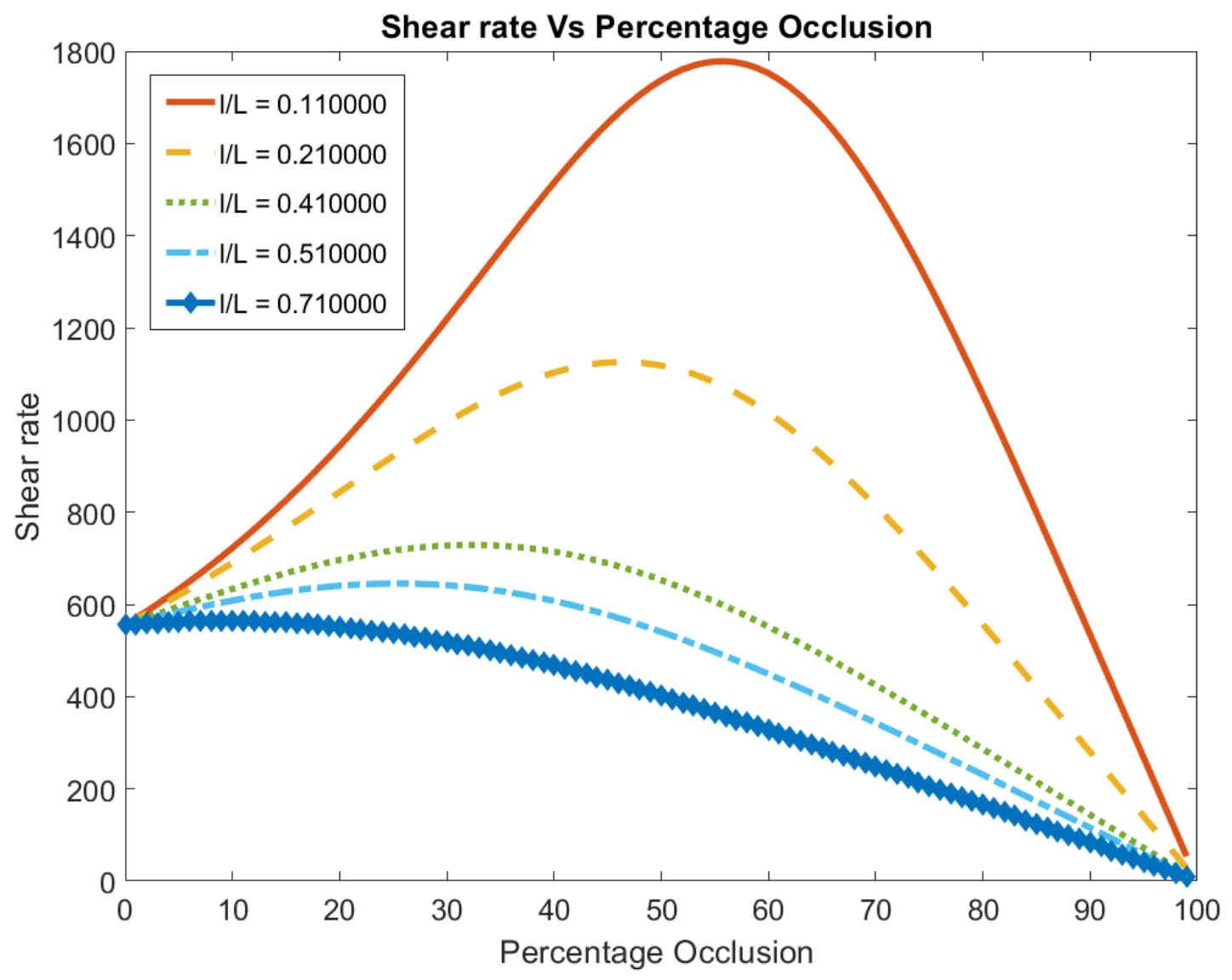

Figure 22 Maximum Shear rate dependency on thrombus length ratio; I/L > 0.5 changes the shear rate behavior from non-linear to monotonically decreasing 


\subsection{Shear rate analysis in a Bifurcation}

Shear rate dynamics on a thrombus growing in a straight channel showed non-monotonic behavior. The longer length of thrombus had an effect on changing this behavior to a monotonically decreasing shear rate. While straight channel was a good way to understanding the shear dynamics involved during thrombus growth, often the geometry of vasculature has an effect on determining the shear dynamics around the thrombus.

Bifurcations of blood vessels are geometries where one blood vessel bifurcates into two branches of varying or equal diameter. Murray's law (Murray, 1926) indicates that blood vessels branch out in different radii such that the energy to transport blood from heart is minimized.

$$
D^{3}=D_{1}{ }^{3}+D_{2}{ }^{3}
$$

D - Diameter of the parent vessel

$D_{1}$ and $D_{2}$ - Diameter of the branches

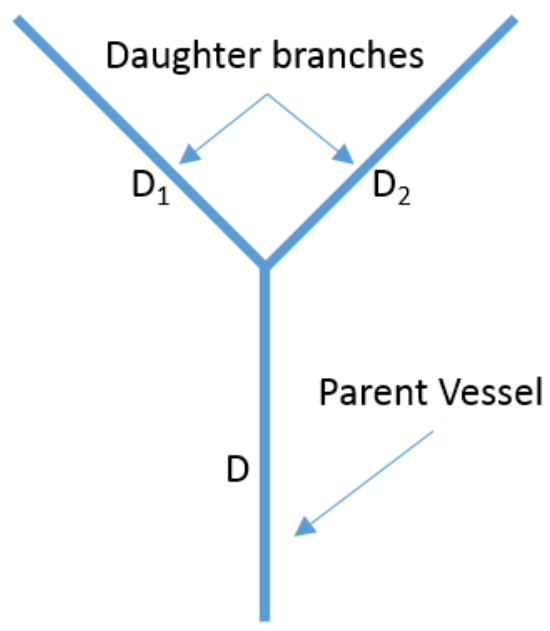

Figure 23 Parent vessel of diameter $D$ bifurcating into two daughter branches with diameters $D_{1}$ and $D_{2}$ 
Bifurcations can be described through bifurcation ratios, dimensionless ratio of diameter of daughter vessels to the parent vessel. Bifurcation ratio is the ratio of daughter diameter to the parent diameter.

$$
r_{1}=\frac{D_{1}}{D}, r_{2}=\frac{D_{2}}{D}
$$

In this study, the concept of critical shear rate has been applied towards the development of a model that relates the maximum shear rate. Each branch is modeled as a series of hydraulic resistances. Resistance with thrombus is increased which increases the overall resistance of the bifurcation. The bifurcation under study is a parallel branch of the circulatory system with the heart pump Increase in bifurcation resistance decreases the flow rate in the bifurcation, effect of flow rate decrease on shear rate is determined by the occlusion percentage of thrombus, radius of bifurcation branches and the percentage of branch length that is covered by thrombus.

Stability of thrombus depends on the evolution of shear rate during the growth of the thrombus. For various diameter ratios, $r_{1}$ and $r_{2}$ the shear rate evolution would change as the initial shear rate would be different for different diameters. For microvasculature bifurcations, diameters in the range of 50-1000 $\mu \mathrm{m}$, the resistances to flow ( $\mathrm{R}_{\text {micro }}$ ) are much higher compared to the vessels of larger diameter $\mathrm{R}_{\text {macro. }}$ When a thrombus grows in microvasculature it adds to the already higher resistance in the microvasculature. This makes the resistance offered by other vessels to flow negligible. All these resistances are similar to the hydraulic resistance discussed in straight channels. 
Heart can be considered as a pump of constant flow rate. When the microvascular resistance increases due to thrombus, the flow gets diverted to other vessels. Belyaev et al. have validated the constant pressure assumption across a microvasculature in their work. In the next section, we describe the model we developed that co-relates bifurcation ratio with the maximum shear rate on the thrombus surface. This model answers the question described in the beginning of chapter 5, in predicting the thrombus that will occlude the vessel.

\subsubsection{Model Development}

Assuming the thrombus to be rectangular and flow to be laminar and newtonian in microvasculature we applied Hagen Poiseuille equation to relate the flow rate and channel resistance. Flow of blood through bifurcation is analogous to flow of current through a circuit of resistances. Figure 24 captures the electrical circuit analogy of bifurcation. The two branches constitute two parallel branches, when a thrombus grows in one of the branches the resistance in that branch increases. This increase in resistance adds to the initial total resistance in the bifurcation. Due to the constant pressure drop boundary condition, the flow rate through bifurcation decreases such that product of flow rate and total resistance $\left(\mathrm{R}_{\mathrm{t}}\right)$ remains constant.

$\mathrm{Q}_{\text {in }}$, the inlet flow rate to the bifurcation splits into $\mathrm{Q}_{1}$ and $\mathrm{Q}_{2}$ into the branches 1 and 2. The flow through each branch is given by the equations (24) and (25)

$$
\begin{aligned}
& Q_{1}=\frac{R_{2}}{R_{1}+R_{2}} Q_{i n} \\
& Q_{2}=\frac{R_{1}}{R_{1}+R_{2}} Q_{i n}
\end{aligned}
$$


$\mathrm{Q}_{1}, \mathrm{Q}_{2}$ - flow rate in branch-1 and branch-2 of bifurcations respectively, $\mathrm{m}^{3} \mathrm{~s}^{-1}$

$\mathrm{R}_{1}, \mathrm{R}_{2}$ - hydraulic resistances, $\mathrm{Pa} \mathrm{s} \mathrm{m}^{-3}$

(a)

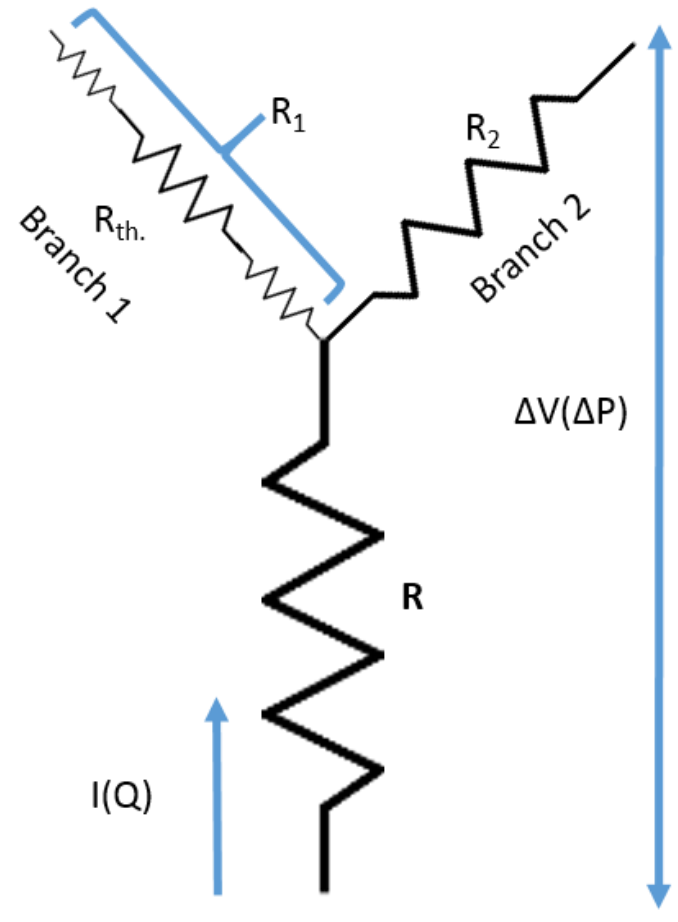

(b)

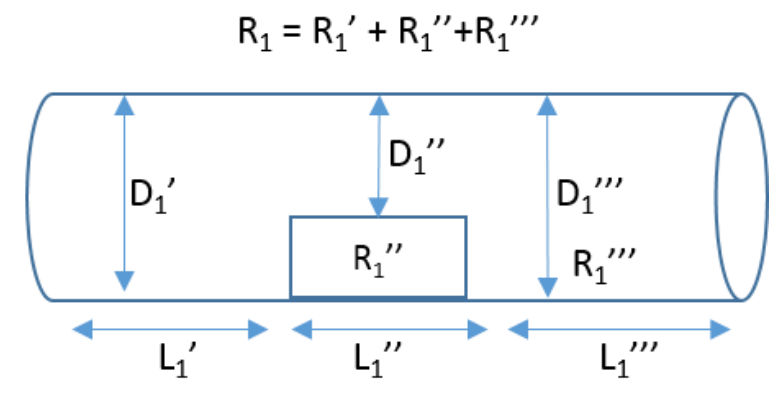

Figure 24 a) Bifurcation as a network of hydraulic resistances, Branch 2 as a single resistance $R_{2}$ and the inlet channel resistance $R$. (b) Branch 1 is the sum of three reistances, $R_{1}^{\prime}, R_{1}^{\prime \prime}, R_{1}{ }^{\prime \prime}$, where $R_{1}{ }^{\prime}$ is the thrombus.

Higher the resistance in branch 2 more of the inlet flow is diverted to branch 1 . The inlet flow rate also depends on both the resistances.

$$
\begin{gathered}
Q=\frac{\Delta P}{R_{t}} \\
R_{t}=R+\frac{R_{1} * R_{2}}{R_{1}+R_{2}}
\end{gathered}
$$


$R_{t}$ is the sum of all the resistances in the bifurcation, i.e. effective resistance of bifurcation. $R_{1}$ and $R_{2}$ are parallel to each other and are connected in series to the parent vessel of resistance $R$. $R_{t}$ is arrived at based on law of addition of parallel and series resistances.

Resistance in branch $1\left(\mathrm{R}_{1}\right)$ is the sum of three resistances.

$$
\begin{gathered}
R_{1}=R_{1}{ }_{1}+R^{\prime \prime}{ }_{1}+R^{\prime \prime \prime}{ }_{1} \\
R_{1}^{\prime}=\frac{128 \mu L_{1}^{\prime}}{\pi\left(D^{\prime}{ }_{1}\right)^{4}}, \quad R^{\prime \prime}{ }_{1}=\frac{128 \mu L^{\prime \prime}{ }_{1}}{\pi\left(D^{\prime \prime}{ }_{1}\right)^{4}}, \quad R^{\prime \prime \prime}{ }_{1}=\frac{128 \mu L^{\prime \prime \prime}{ }_{1}}{\pi\left(D^{\prime \prime \prime}{ }_{1}\right)^{4}}
\end{gathered}
$$

$D^{\prime}{ }_{1}$ and $D^{\prime \prime \prime}{ }_{1}$ are the diameters of vessel that don't change during thrombus growth, while $D^{\prime \prime}{ }_{1}$ decreases from the initial diameter $\mathrm{D}_{1}$ at $0 \%$ percent occlusion to 'zero' at $100 \%$ occlusion.

The shear rate on the thrombus surface $\dot{\gamma_{1}}$ is shear rate at the occluded region with diameter $D^{\prime \prime}{ }_{1}$ and $\mathrm{Q}_{\text {in }}$ is the inlet flow rate to the bifurcation.

$$
\begin{gathered}
\dot{\gamma_{1}}=\frac{32 * Q_{\text {in }} * \lambda_{2}}{\left(\lambda_{1}+\lambda_{2}\right) * \pi *\left(D^{\prime \prime}{ }_{1}\right)^{3}} \\
\lambda_{1}=\frac{L_{1}^{\prime}}{\left(D^{\prime}{ }_{1}\right)^{4}}+\frac{L^{\prime \prime}{ }_{1}}{\left(D^{\prime \prime}{ }_{1}\right)^{4}}+\frac{L^{\prime \prime \prime}{ }_{1}}{\left(D^{\prime \prime \prime}{ }_{1}\right)^{4}}, \quad \lambda_{2}=\frac{L_{2}}{\left(D_{2}\right)^{4}}
\end{gathered}
$$

The shear rate on the thrombus surface during its growth in branch-1in terms of the bifurcation ratio and occlusion ratio in terms of shear rate at the bifurcation inlet and the occlusion percentage is given by equation (32). Derivation of these equations can be seen in appendix. 


$$
\begin{gathered}
\dot{\gamma}_{1}=\frac{\lambda_{2} * \dot{\gamma}}{\left(r_{1}{ }^{3} *\left(1-x_{1}\right)^{3}\right) *\left(\lambda_{1}+\lambda_{2}\right)} \\
x_{1}=1-\frac{D_{1}{ }^{\prime \prime}}{D_{1}}
\end{gathered}
$$

$\dot{\gamma}-$ Shear rate in the parent vessel, $\mathrm{s}^{-1}$

$\dot{\gamma}_{1}-$ Shear rate in branch- 1 of bifurcation, $\mathrm{s}^{-1}$

$x_{1}$ - Occlusion ratio in branch-1, $100 x_{1}$ - occlusion percentage

Parameter $\lambda_{1}$ still carries the length of the thrombus $L^{\prime \prime}{ }_{1}$ which makes the equation harder to analyze in terms of just $\mathrm{x}_{1}, \mathrm{r}_{1}$ and $\mathrm{r}_{2}$. To make the analysis simpler, the length of thrombus is expressed in terms of a dimensionless quantity ' $\Phi$ ' and the total length of the branch $\mathrm{L}_{1}$. ' $\Phi$ ' represents the ratio of length of thrombus to the length of the branch itself. This enables us to compare thrombi of different lengths in bifurcations of various sizes.

$$
\Phi=\frac{L_{1}^{\prime \prime}}{L_{1}}
$$

Now the shear rate equation can be refined with the substitution of $\Phi$ in $\lambda_{1}$ of equation (32) and simplified further to yield equation (34)

$$
\dot{\gamma}_{1}=\frac{\lambda_{2} * \dot{\gamma}}{\left(r_{1}^{3} *\left(1-x_{1}\right)^{3}\right) *\left(\lambda_{1}+\lambda_{2}\right)} ; \lambda_{1}=\frac{L_{1}}{r_{1}^{4} D^{4}}\left[(1-\Phi)+\frac{\Phi}{\left(1-x_{1}\right)^{4}}\right]
$$


The main interest lies in the maximum shear rate during thrombus growth. Keeping the injury length ratio, pressure drop and other parameters constant we can investigate the relation between the maximum shear rate and the occlusion ratio as the thrombus grows. The occlusion ratio at which the maximum shear rate is defined as the critical occlusion ratio $\left(x_{1}^{*}\right)$. Critical occlusion ratio is the value of $x_{1}$ at which the derivative of shear rate function in equation (34) becomes zero. Equation (35) captures the critical occlusion ratio in terms of $r_{1}, r_{2}$ and

$$
\mathrm{x}_{1}{ }^{*}=1-\left\{\frac{\mathrm{L}_{1} * \mathrm{~L} * \Phi * \mathrm{r}_{2}{ }^{4}+\Phi * \mathrm{~L}_{1} * \mathrm{~L}_{2}}{\mathrm{~L}_{1} * \mathrm{~L}_{2} *(1-\Phi)+\mathrm{L} * \mathrm{~L}_{1} *(1-\Phi) * \mathrm{r}_{2}{ }^{4}+\mathrm{L} * \mathrm{~L}_{2} * \mathrm{r}_{1}{ }^{4}}\right\}^{1 / 4}
$$

See the appendix for the development of these equations.

$\mathrm{L}_{1}$ - Total length of branch-1

$\mathrm{L}_{2}-$ Total length of branch-2

L - Length of parent vessel

$\Phi=\frac{L_{1}^{\prime \prime}}{L_{1}}-$ Ratio of length of thrombus to the total length of branch-1

$\dot{\gamma}$ - Shear rate at the parent vessel

There are four roots for equation (35), but only one real root in the range of 0 to 1 . Practically occlusion ratio cannot be $>1$, so the solutions of $\mathrm{x}_{1}{ }^{*}>1$ are ignored. Critical occlusion ratio was applied in (34) to obtain the maximum shear rate on the thrombus for each case.

Equations (34) and (35) allows one to study the effect of different $r_{1}$ and $r_{2}$, for a thrombus of constant length growing under a constant pressure drop, on the maximum shear rate during 
thrombus growth. If this maximum shear rate exceeds the critical shear rate, $3400 \mathrm{~s}^{-1}$ in this case, then the hydrodynamic forces would disrupt the occlusion of thrombus.

Murray's law can be stated in other terms, as the sum of cube of bifurcation ratios is one. Dividing equation (22) by $\mathrm{D}^{3}$ on the both sides yields,

$$
1=\left(\frac{D_{1}}{D}\right)^{3}+\left(\frac{D_{2}}{D}\right)^{3}=r_{1}{ }^{3}+r_{2}{ }^{3}
$$

Equation (36) is another way of looking at Murray’s law as per which the bifurcation ratios cannot be greater than 1 as this would lead to sum of cubes of bifurcation ratios more than 1 . The study was limited to bifurcation ratios in the range of 0.1 to 1 as per Murray's law.

\subsubsection{Bifurcation ratio and Thrombus Stability}

The maximum shear rate during thrombus growth is a function of ratio of bifurcation $r_{1}$ and $r_{2}$, length of the injury and diameter of the parent vessel. The branch without the thrombus is analogous to a shunt resistance in the electrical circuit. A new dimensionless shear ratio is defined to make the comparison simpler and easier to comprehend.

Shear ratio is defined as the ratio of maximum shear rate during thrombus growth to the critical shear rate, $3400 \mathrm{~s}^{-1}$ in this case. Shear ratio is a function of thrombus length ratio, $\Phi$, bifurcation ratios, $\mathrm{r}_{1}$ and $\mathrm{r}_{2}$, and the shear rate at the inlet channel $\dot{\gamma}$. 


$$
\text { Shear ratio }=\frac{\left(\dot{\gamma}_{1}\right)_{\max }}{\dot{\gamma}_{c}}=\frac{\left(\dot{\gamma}_{1}\right)_{\max }}{\gamma_{c}}=\frac{\lambda_{2} * \dot{\gamma}}{\left(r_{1}{ }^{3} *\left(1-x_{1}{ }^{*}\right)^{3}\right) *\left(\lambda_{1}+\lambda_{2}\right)}=f\left(\Phi, r_{1}, r_{2}, \dot{\gamma}\right)
$$

The shear rate at the inlet by itself is a function of thrombus length, pressure and the diameter of the parent vessel. Overall, the shear ratio is dependent only on the bifurcation ratio, injury length ratio, pressure and the diameter of the parent vessel. Keeping the injury length ratio and pressure constant in the model, the relationship between $r_{1}, r_{2}$ and shear ratio was investigated. Figure 23 represents this relationship for $\Phi=0.021, \mathrm{D}=38 \mu \mathrm{m}$ and $\Delta \mathrm{P}=100 \mathrm{~Pa}$.

For each $r_{1}$, the shear ratio for all $r_{2}$ were simulated in MATLAB and the phase plot in Figure 23 was generated.

A red dotted line at the shear ratio $=1$, segregates the plot into two regions of partial occlusion and complete occlusion. The set of $r_{1}$ and $r_{2}$ values that lie above the red line correspond to bifurcations with a stable thrombus with partial occlusion for this configuration of diameter and pressure. For example, when $r_{1}=0.2$, all $r_{2}=0.1$ to 0.9 all the shear ratios fall under the complete occlusion region. In these bifurcations, the initial shear rate is the maximum shear rate and as the thrombus grows the shear rate decreases monotonically.

If the ratio of maximum shear rate to the critical shear rate exceeds one, the thrombus will not occlude the vessel. This is true for various configurations of $r_{1}$ and $r_{2}$ that is evident from the phase plot. As the $r_{2}$ value increases the possible $r_{1}$ values for complete occlusion also increase. When $r_{2}$ is 0.9 (orange dotted line in Figure 26), for any $r_{1}$ from 0.1 to 0.7 , the thrombus is completely occluding, whereas for $\mathrm{r}_{2}=0.1, \mathrm{r}_{1}$ has a smaller range of 0.1 to 0.4 for complete occlusion. When $\mathrm{r}_{2}$ is larger, the resistance in branch 2 is less, which implies only a longer thrombus can increase the total resistance of the bifurcation to minimize the shear forces around the thrombus as it grows. 


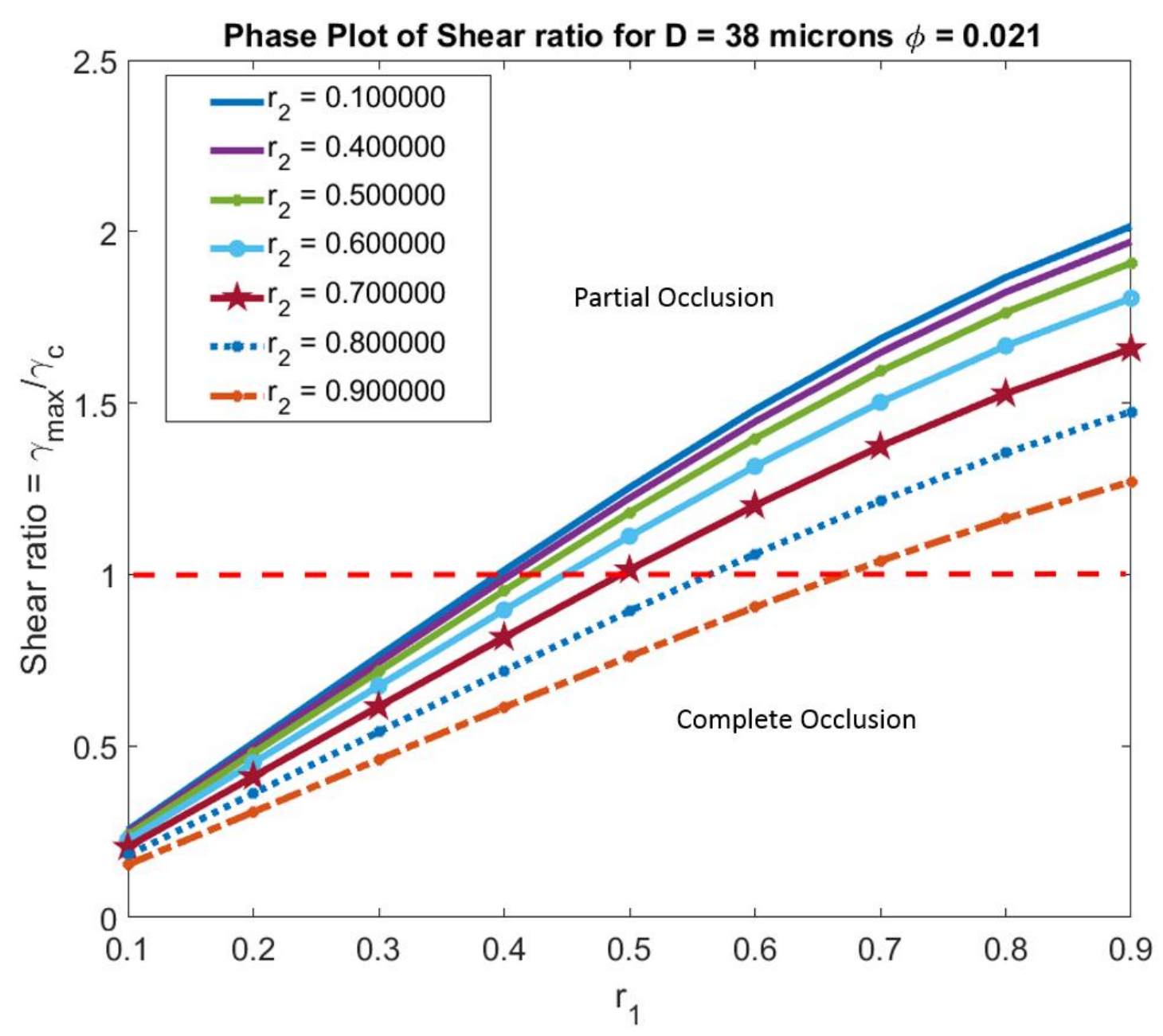

Figure 25 Bifurcation ratios $r_{1}, r_{2}$ and Shear ratio, $\gamma_{\max } / \dot{\gamma}_{c}$ plot for $\emptyset=0.021$ and an inlet diameter $D=38 \mu \mathrm{m}$

Figure 25 is a phase plot for $\mathrm{D}=38 \mu \mathrm{m}$ and $\Phi=0.021$, i.e. the thrombus length is $2.1 \%$ of the length of branch-1. For each $\mathrm{r}_{1}$, the shear ratio for all $\mathrm{r}_{2}$ values were obtained using equation (37) and plotted against $\mathrm{r}_{1}$ using MATLAB®. Portions of line that lie above the red-dotted line indicate the bifurcation ratios that will have a stable thrombus. The points in the plot lying below the shear ratio $=1$, correspond to bifurcation geometries with $100 \%$ occlusion for thrombi of length ratio 0.021 . 
For different thrombus length ratios, new phase plots can be generated from the model relating all $r_{1}$ and $r_{2}$ values against the shear ratio. Critical shear rate used in the model is the estimated critical shear rate for rat vasculature. The same mathematical model can be applied to investigate the bifurcation geometries of human vasculature with the corresponding critical shear rate estimated using experimental data on human blood. 


\section{Conclusion}

Geometry plays a vital role in determination of thrombogenic sites. An integrated COMSOL model was developed to predict the sites of possible thrombus generation in ladder network. The existing knowledge on triggers for thrombus formation has been integrated in the ladder network model. Results of simulation and experiment showed the importance of geometry in the formation and stability of thrombus. The results of prediction from the model agreed well with the experiments conducted on the microfluidic device using whole human blood. Thrombi were assumed as spherical, non-porous structures that have a constant surface concentration of thrombin in COMSOL model to understand and predict the patterns of thrombus formation in ladder network. Stagnation points, shear gradient, convection of thrombin and concentration of platelets were found to be the primary metrics that controlled thrombus nucleation in ladder network. The integrated model gave preliminary predictions of thrombus nucleation sites based on the metrics discussed in the study. Results from the model could be improved with the inclusion of deformability associated with blood cells. In certain geometries cell deformation is an important factor for thrombus nucleation (E.C.Eckstein \& F.Belgacem, 1991). The current model does not include the inactivation of thrombin by plasma inhibitors. Thrombin inactivation due to antithrombin III and heparin cofactor II is reported to prevent formation of thrombus downstream, in literature. Creeping flow condition was crucial to the model solution, which limits the usage of the model to $R e<1$. Flows with higher $R e$, need the model to consider the effects of inertial force in hemodynamics. Results from the integrated COMSOL model was published in Cellular and Molecular Bioengineering (Zilberman, Sylman, Lakshmanan, J.T.McCarty, \& Maddala, 2017) and researchers are already citing this work in their research. 
Geometry plays an important role in determining hydrodynamic forces around a thrombus. The results from COMSOL model were analyzed to understand the effect of thrombi in first few bypasses on the shear gradient, platelet numbers and thrombin convection in other bypasses. The evolution of shear rate during thrombus growth is dependent on the size of the thrombus, geometry and the boundary conditions. For a constant inlet flow rate, thrombus in the bypasses experienced a non-linear shear rate during growth.

Shear forces around a thrombus undergo non-linear increase and decrease under constant pressure drop boundary condition. In a straight channel, longer thrombus experiences lesser shear forces during its growth causing it to grow and occlude the vessel completely vis-à-vis a shorter thrombus has higher shear forces around causing it to stabilize before complete occlusion. The importance of injury length in determining the occlusion scenario of a thrombus was explained through critical shear rate in the literature (Belyaev, Panteleev, \& Ataullakhanov, 2015).

Importance of bifurcation ratios in terms of energy minimization is the idea behind Murray's law. Blood vessel bifurcation ratios in terms of thrombus stability was investigated in this study using critical shear rate. The phase plot shows how bifurcation ratio can determine the thrombus stability in microvasculature. A thrombus growing in any of the bifurcation branches increases the resistance in the bifurcation. The increase in resistance lowers the flow rate around the thrombus. Shear forces decrease along with decrease in flow rate depending on the initial shear rate in the bifurcation. Initial shear rate in the bifurcation is dependent on the length of the thrombus, radius of bifurcation branches and pressure. Thrombi in ladder network occluded the bypasses to different extents based on the shear forces around the thrombus. The thrombus growing near the outlet channel was shielded from the shear disturbances by the thrombus near the inlet channel. This is an example of an evolving geometry's impact on the occlusion of thrombus. As a geometry evolves 
the flow direction and velocity of blood changes. The changes in the geometry affect the transport of thrombin and platelets to thrombus affecting its growth rate. The current work shows that the geometry of vasculature plays an important role in determining thrombus nucleation, growth and stability. Integrated approaches like these could result in new patient specific metrics to determine the risk of Cardiovascular diseases. 


\section{Future Work}

The potential of the integrated model developed to identify thrombus nucleation sites through quantifiable metrics is immense. Simulations could be repeated under constant pressure conditions to elucidate the importance of boundary conditions on thrombus formation and also to prove the versatility of the model. The metrics developed in the work could be quantified through experiments on specially designed devices that can measure the parameters involved in thrombus formation. Quantified metrics can be used to design an optimized geometry with minimal thrombus formation. Current ECMOs involve the use of heparin, an anticoagulant to prevent blood clots in the devices. With an improved design, that minimizes the geometrical features that support thrombus formation, the use of heparin could be minimized. Monitoring a patient's ability to form clots is important for the diagnosis of bleeding disorders. Diagnostic device could be designed based on the integrated model to analyze patient's blood for clotting. The approach used in the model development can be extrapolated to study the vascular beds in lungs and brain. Geometrical effects on thrombus formation can be used to design diagnostic devices for vWF deficiency. The theory on bifurcation ratio controlling the occlusion scenario of thrombus can be verified through experiments under different pressure conditions. In-vivo data on primates and mice can be used to validate the bifurcation ratio theory on thrombus stability. Theory on thrombus stability can be extended to more branched geometries like ladder through experiments and simulations. 


\section{Appendix}

The resistances in Figure 25 are given by equation (29) and (30). Total resistance of bifurcation is given by

$$
\begin{gathered}
R_{t}=R+\frac{R_{1} * R_{2}}{R_{1}+R_{2}} \\
R_{1}=\frac{128 \mu L^{\prime}{ }_{1}}{\pi\left(D^{\prime}{ }_{1}\right)^{4}}+\frac{128 \mu L^{\prime \prime}{ }_{1}}{\pi\left(D^{\prime \prime}{ }_{1}\right)^{4}}+\frac{128 \mu L^{\prime \prime \prime}{ }_{1}}{\pi\left(D^{\prime \prime \prime}{ }_{1}\right)^{4}} \\
R_{1}=\frac{128 \mu}{\pi}\left(\frac{L_{1}^{\prime}}{\left(D^{\prime}{ }_{1}\right)^{4}}+\frac{{L^{\prime \prime}}_{1}}{\left(D^{\prime \prime}{ }_{1}\right)^{4}}+\frac{L^{\prime \prime \prime}{ }_{1}}{\left(D^{\prime \prime \prime}{ }_{1}\right)^{4}}\right)=\frac{128 \mu}{\pi} \lambda_{1} \\
R_{2}=\frac{128 \mu L_{2}}{\pi\left(D_{2}\right)^{4}}=\frac{128 \mu}{\pi} \lambda_{2} \\
\frac{R_{1} * R_{2}}{R_{1}+R_{2}}=\frac{128 \mu}{\pi}\left(\frac{\lambda_{1} * \lambda_{2}}{\lambda_{1}+\lambda_{2}}\right)
\end{gathered}
$$

To simplify the length and diameter terms in the reistance they are grouped under variables $\lambda_{1}$ and $\lambda_{2}$ for $R_{1}$ and $R_{2}$ respectively in equations (A-3) and (A-4). The only variable in $\lambda_{1}$ that changes during thrombus growth is $D^{\prime \prime}{ }_{1}$ while the rest of the parameters remain constant for a given bifurcation, with the constant thrombus length.

The total reistance of the bifurcation is given by

$$
R_{t}=\frac{128 \mu L}{\pi D^{4}}+\frac{128 \mu}{\pi}\left(\frac{\lambda_{1} * \lambda_{2}}{\lambda_{1}+\lambda_{2}}\right)=\frac{128 \mu}{\pi}\left(\lambda+\frac{\lambda_{1} * \lambda_{2}}{\lambda_{1}+\lambda_{2}}\right)
$$


Equation (A-6) gives the total resistance of the bifurcation that can be updated for the change in diameter as the thrombus grows and used in equation (26) for determining the flow rate through bifurcation.

Assuming laminar flow in the bifurcation, the shear rate on the thrombus surface is given by velocity near the thrombus and the diameter of vessel $\mathrm{D}_{1}{ }^{\prime}$.

$$
\dot{\gamma_{1}}=\frac{8 * v^{\prime \prime}{ }_{1}}{D^{\prime \prime}{ }_{1}} ; \quad v^{\prime \prime}{ }_{1}=\frac{Q_{1}}{A^{\prime \prime}{ }_{1}}=\frac{R_{2}}{A^{\prime \prime}{ }_{1}\left(R_{1}+R_{2}\right)} Q_{\text {in }} ; A^{\prime \prime}{ }_{1}=0.25 \pi\left(D^{\prime \prime}{ }_{1}\right)^{2}
$$

$v^{\prime \prime}{ }_{1}$ - Velocity near thrombus, i.e velocity in resistance $R^{\prime \prime}{ }_{1}$.

$A^{\prime \prime}{ }_{1}$ - cross sectional area of resistance $R^{\prime \prime}{ }_{1}$

$$
Q_{\text {in }}=\frac{\Delta P}{R_{t}}=\frac{\Delta P}{\frac{128 \mu}{\pi}\left(\lambda+\frac{\lambda_{1} * \lambda_{2}}{\lambda_{1}+\lambda_{2}}\right)}=\frac{\Delta P * \pi *\left(\lambda_{1}+\lambda_{2}\right)}{128 \mu\left(\lambda\left(\lambda_{1}+\lambda_{2}\right)+\lambda_{1} * \lambda_{2}\right)}
$$

$R_{1}$ and $R_{2}$ from equation (A-4) and (A-3) substituted into equation (A-7) gives the velocity near the thrombus surface.

$$
v^{\prime \prime}{ }_{1}=\frac{4 Q_{\text {in }}}{\pi\left(D^{\prime \prime}{ }_{1}\right)^{2}} \frac{\lambda_{2}}{\lambda_{1}+\lambda_{2}}=\frac{4}{\pi\left(D^{\prime \prime}{ }_{1}\right)^{2}} * \frac{\Delta P * \pi *\left(\lambda_{1}+\lambda_{2}\right)}{128 \mu\left(\lambda\left(\lambda_{1}+\lambda_{2}\right)+\lambda_{1} * \lambda_{2}\right)} * \frac{\lambda_{2}}{\lambda_{1}+\lambda_{2}}
$$

Equation (A-9) is an unreduced version of the velocity equation that can be used to determine the shear rate on the thrombus surface. Substituting (A-9) in (A-7) and reducing it further yields equation $(\mathrm{A}-10)$. 


$$
\dot{\gamma_{1}}=\frac{\lambda_{2} * \Delta P}{4 \mu\left(D^{\prime \prime}{ }_{1}\right)^{3}\left(\lambda\left(\lambda_{1}+\lambda_{2}\right)+\lambda_{1} \lambda_{2}\right)}
$$

Equation (A-10) gives the shear rate on the thrombus surface in terms of known quanitites that can be updated based on thrombus growth. The change in occlusion percentage changes the $D^{\prime \prime}{ }_{1}$ and $\lambda_{1}$ as per equation (33) while the other quantities in the equation are constants.

Equation (A-10) is rewritten in terms of occlusion ratio,

$$
D^{\prime \prime}{ }_{1}=D_{1}\left(1-x_{1}\right) ; \lambda_{1}=\frac{L_{1}^{\prime}}{D_{1}^{\prime 4}}+\frac{L_{1}^{\prime \prime}}{\left(D_{1}\left(1-x_{1}\right)\right)^{4}}+\frac{L^{\prime \prime \prime}{ }_{1}}{D_{1}^{\prime \prime \prime}}
$$

The diameters $D^{\prime}{ }_{1}$ and $D^{\prime \prime \prime}{ }_{1}$ remain unchanged during thrombus growth and are equal to $D_{1}$ the initial diameter of branch 1 .

$$
\lambda_{1}=\frac{L_{1}^{\prime}+L^{\prime \prime \prime}{ }_{1}}{D_{1}^{4}}+\frac{L^{\prime \prime}{ }_{1}}{\left(D_{1}\left(1-x_{1}\right)\right)^{4}} ; \lambda_{2}=\frac{L_{2}}{D_{2}^{4}} ; \lambda=\frac{L}{D^{4}}
$$

Dimensionless variables in an equation make it easier to comprehend and compare the solutions. Parameters $\lambda_{1}$, and $\lambda_{2}$ can be rewritten in terms of bifurcation ratio $r_{1}$ and $r_{2}$ as 


$$
\lambda_{1}=\frac{L_{1}^{\prime}+L^{\prime \prime \prime}{ }_{1}}{r_{1}^{4} D^{4}}+\frac{L^{\prime \prime}{ }_{1}}{\left(r_{1}^{4} D^{4}\left(1-x_{1}\right)^{4}\right)} ; \lambda_{2}=\frac{L_{2}}{r_{2}^{4} D^{4}}
$$

Substituting the parameters from (A-13) into (A-10) and simplifying we obtain the following relation for shear rate on thrombus surface in terms of bifurcation ratio, occlusion ratio and pressure drop.

$$
\begin{gathered}
\dot{\gamma_{1}}=\frac{L_{2} r_{1} \Delta P\left(1-x_{1}\right) D}{4 \mu\left[L\left\{A r_{2}^{4} D^{4}+L^{\prime \prime}{ }_{1} r_{2}^{4} D^{4}+L_{2} r_{1}^{4}\right\}+L_{2}\left\{A+L_{2} L^{\prime \prime}{ }_{1}\right\}\right]} ; \\
A=\left(L^{\prime}{ }_{1}+L^{\prime \prime \prime}{ }_{1}\right)\left(1-x_{1}\right)^{4}
\end{gathered}
$$

Equation (A-14) is the shear rate on the thrombus surface in branch 1 expressed in dimensionless quantities of occlusion ratio and bifurcation ratio along with other parameters such as $\mathrm{D}$, pressure drop, $\mathrm{r}_{2}$, lengths of the thrombus $\left(L^{\prime \prime}{ }_{1}\right)$, and vessel $(L)$ and blood viscosity $(\mu)$.Differentiating equation (A-14) with respect to $\mathrm{x}_{1}$ and finding the roots of the derivative would yield the critical occlusion ratio mentioned in equation (35). 


\section{References}

1. Belyaev, A. V., Panteleev, M. A., \& Ataullakhanov, F. I. (2015). Threshold of Microvascular Occlusion: Injury Size Defines the Thrombosis. Biophysical Journal, 450456.

2. Boyer, C. J., \& Swartz, R. D. (1991). Severe clotting during extracorporeal dialysis procedures. Seminars in Dialysis, 69-71.

3. Colace, T., G.W.Tormoen, O.J.T.McCarty, \& S.L.Diamond. (2013). Microfluidics and coagulation biology . Annu. Rev. Biomed. Eng., 283-303.

4. Falati, S. P. (2002). Real-time in vivo imaging of platelets, tissue factor and fibrin during arterial thrombus formation. Nat. Med, 1175-1181.

5. Fogelson. (2015). Fluid mechanics of blood clot formation. Annual Review of Fluid Mechanics, 377-403.

6. Harmening, D. M. (2002). Clinical Hematology and Fundamentals of Hemostasis, 4th Edition. F.A.Davis Company.

7. Hussain, M. A. (1999). Relationship between power law coefficients and major blood constituents affecting the whole blood viscosity. J. Biosci, 329-337.

8. J.J, H. (2006). Flow effects on coagulation and thrombosis. Arterioscler. Thromb. Vasc. Biol, 1729-1737.

9. Murray, C. (1926). The physiological principle of minimum work. I. The vascular system and the cost of blood volume. Proceedings of the National Academy of Science USA, 207214.

10. Nesbitt, W. S. (2009). A shear gradient-dependent platelet aggregation mechanism drives thrombus formation. Nat. Medicine, 665-673.

11. Okorie, U. M. (2008). Determination of surface tissue factor thresholds that trigger coagulation at venous and arterial shear rates: amplification of $100 \mathrm{fM}$ circulating tissue factor requires flow. Blood, 3507-3513.

12. R.B., B., Stewart, W., \& E.N.Lightfoot. (1960). Transport Phenomena. New York: Wiley.

13. Sato, M., \& Ohshima, N. (1986). Hemodynamics at Stenoses Formed by Growing Platelet Thrombi in Mesenteric Microvasculature of Rat. Mircovascular Research, 66-76. 
14. Stalker, T., E.A.Traxler, \& L.F.Brass. (2013). Hierarchial organization in the hemostatic response and its relationship to the plateletsignaling network. Blood, 1875-1885.

15. Thomas V. Colace, R. W. (2012). Thrombus Growth and Embolism on Tissue FactorBearing Collagen Surfaces Under Flow. Arteriosclerosis, Thrombosis, and Vascular Biology, 1466-1476.

16. Tokarev, A., Butylin, A., \& Ataullakhanov.F.I. (2011). Platelet adhesion from shear blood flow is controlled by near-wall rebounding collisions with erythrocytes. Biophysics Journal, 799-808.

17. Westein, E. A.-P. (2013). Atherosclerotic geometries exacerbate pathological thrombus formation poststenosis in a vonWillebrand factor-dependent manner. Proc. Natl. Acad. Sci., 1357-1362.

18. Wu, W. (2013). Lung assist device: development of microfluidic oxygenators for preterm infants with respiratory failure. Lab Chip, 2641-2650.

19. Zilberman, J., Sylman, J., Lakshmanan, H. H., J.T.McCarty, O., \& Maddala, J. (2017). Dynamics of Blood Flow and Thrombus Formation in a Multi-Bypass. Cellular and Molecular Bioengineering, 16-29. 